ARTICLE

\title{
Tumors induce de novo steroid biosynthesis in $T$ cells to evade immunity
}

Bidesh Mahata (1,2凶, Jhuma Pramanik², Louise van der Weyden², Krzysztof Polanski (iD ${ }^{2}$, Gozde Kar ${ }^{3,8}$,

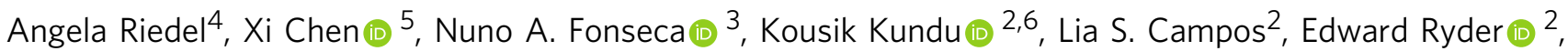
Graham Duddy², Izabela Walczak ${ }^{2}$, Klaus Okkenhaug (iD ${ }^{1}$, David J. Adams (D) ${ }^{2}$, Jacqueline D. Shields (i) ${ }^{4 凶} \&$ Sarah A. Teichmann (10) $2,7 凶$

Tumors subvert immune cell function to evade immune responses, yet the complex mechanisms driving immune evasion remain poorly understood. Here we show that tumors induce de novo steroidogenesis in T lymphocytes to evade anti-tumor immunity. Using a transgenic steroidogenesis-reporter mouse line we identify and characterize de novo steroidogenic immune cells, defining the global gene expression identity of these steroidproducing immune cells and gene regulatory networks by using single-cell transcriptomics. Genetic ablation of $T$ cell steroidogenesis restricts primary tumor growth and metastatic dissemination in mouse models. Steroidogenic T cells dysregulate anti-tumor immunity, and inhibition of the steroidogenesis pathway is sufficient to restore anti-tumor immunity. This study demonstrates $T$ cell de novo steroidogenesis as a mechanism of anti-tumor immunosuppression and a potential druggable target.

\footnotetext{
${ }^{1}$ Department of Pathology, University of Cambridge, Cambridge CB2 1QP, UK. ${ }^{2}$ Wellcome Sanger Institute, Wellcome Genome Campus, Hinxton, Cambridge CB10 1SA, UK. ${ }^{3}$ EMBL-European Bioinformatics Institute, Wellcome Genome Campus, Hinxton, Cambridge CB10 1SD, UK. ${ }^{4}$ Medical Research Council Cancer Unit, Hutchison/Medical Research Council Research Centre, Cambridge, UK. ${ }^{5}$ Department of Biology, Southern University of Science and Technology, Shenzhen, China. ${ }^{6}$ Department of Haematology, University of Cambridge, Cambridge Biomedical Campus, Long Road, Cambridge CB2 OPT, UK. 7 Theory of Condensed Matter, Cavendish Laboratory, $19 \mathrm{JJ}$ Thomson Ave, Cambridge CB3 OHE, UK. ${ }^{8}$ Present address: Translational Medicine, Research and Early Development, Oncology R\&D, AstraZeneca, Cambridge, United Kingdom.®email: bm562@cam.ac.uk; JS970@mrc-cu.cam.ac.uk; st9@sanger.ac.uk
} 
S teroidogenesis is a metabolic process by which cholesterol is converted to steroids ${ }^{1}$. The biosynthesis of steroids starting from cholesterol is often termed de novo steroidogenesis 1 . Cytoplasmic cholesterol is transported into the mitochondria, where the rate-limiting enzyme CYP11A1 (also known as P450 side chain cleavage enzyme) converts cholesterol to pregnenolone. Pregnenolone is the first bioactive steroid of the pathway, and the precursor of all other steroids (Fig. 1a) ${ }^{1,2}$. The steroidogenesis pathway has been extensively studied in adrenal gland, gonads, and placenta. De novo steroidogenesis by other tissues, known as extra-glandular steroidogenesis, in brain ${ }^{1,3}$, skin $^{4}$, thymus ${ }^{5}$, and adipose tissues ${ }^{6}$ has also been reported. Steroid production as a result of immune response in the mucosal tissues, such as in the lung and intestine, has been shown to play a tolerogenic role to maintain tissue homeostasis ${ }^{7,8}$. However the physiological and pathological role of extra-glandular steroidogenesis remains largely unknown ${ }^{2}$.

In cancer, the immunosuppressive tumor microenviroenmnt (TME) prevents immune cells from mounting an effective antitumor immune response ${ }^{9}$. Several mechanisms by which cancer cells evade the immune system have been described ${ }^{10,11}$. These include: (1) immune suppression at the TME mediated by immunosuppressive cells, (2) induction of apoptosis in cytotoxic T-lymphocytes (CTLs) by the expression of pro-apoptotic ligands e.g., Fas ligand and TRAIL, (3) dysregulating antigen presentation, (4) release of immunosuppressive factors such as IL-10 and TGF $\beta$, and (5) inducing tolerance and immune deviation by mechanisms including, among others, shifting the balance of Th1 immune responses (type-1 immune response) to Th2 (type-2 immune response), and expression of immune inhibitory molecules such as PD-1 (programmed death-1) and CTLA-4 (CTL antigen-4). Established tumor often show type 2 immune responses that drive an immunocompromised and pro-tumorigenic program ${ }^{12-25}$. Further understanding of the anti-tumor immunosuppression would allow us to develop immunotherapies.

Steroid hormones are known immunosuppressive biomolecules ${ }^{26,27}$. We recently reported that type $2 \mathrm{CD}^{+} \mathrm{T}$ cells (Th2 lymphocytes) induce de novo steroidogenesis to restore immune homeostasis by limiting the immune response against a worm parasite $\mathrm{e}^{28}$. Thus we sought to determine whether type 2 $\mathrm{T}$ cell-mediated steroidogenesis contributes to the generation of a suppressive niche in the TME.

To study de novo steroidogenesis we generated two transgenic mouse lines: a fluorescent reporter mouse line (Cyp11a1mCherry) and a conditional Cyp11a1 (floxed) knockout mouse line. We show the presence of de novo steroidogenesis by tumorinfiltrating $\mathrm{T}$ lymphocytes, but not in unchallenged animals or draining lymph nodes. Genetic ablation of Cyp11a1 in T cells restricts experimental primary tumor growth and lung metastasis. Mechanistically, we find that intratumoral $\mathrm{T}$ cell steroidogenesis dysregulates anti-tumor immunity that could be restored by inhibiting the steroidogenesis pathway pharmacologically. This study therefore demonstrates that $\mathrm{T}$ cell de novo setroidogenesis is a cause of anti-tumor immunosuppression and a potential drug target for cancer immunotherapy.

\section{Results}

Generation of Cyp11a1 reporter and conditional knockout mice. Cyp11a1 is the first and rate-limiting enzyme during steroid production. The expression of Cyp11a1 is therefore also a faithful biomarker of de novo steroidogenesis ${ }^{1}$. Therefore, we generated a reporter mouse line to identify Cyp11a1-expressing steroidogenic cells definitively (Fig. 1b, c, Supplementary Fig. 1a-d). As expected, mCherry expression was detected in single-cell suspensions of testis and adrenal glands but negligible to no expression in the spleen (Fig. 1c) or other tissues including lung, kidney, blood, liver, bone marrow, lymph nodes, and thymus (Supplementary Fig. 1b). However, Cyp11a1-mCherry signal was detected specifically in activated type- $2 \mathrm{CD} 4^{+} \mathrm{T}$ helper cells (Th2 cells) upon activation in vitro (Supplementary Fig. 1c), as reported previously ${ }^{28}$. Cyp11a1 expression was detectable only in mCherry-expressing $\mathrm{T}$ helper cells (Supplementary Fig. 1d).

To determine the functional consequences of cell-type-specific steroidogenesis we created a Cyp11al floxed (Cyp11a1 flfl $)$ mouse following EUCOMM/WSI conditional gene targeting strategy ${ }^{29}$. Briefly, a knockout-first (tmla) mouse line was created using a promoter-driven targeting cassette (Fig. 1d). The tm 1 a mouse was then crossed with Flp-deleter mice (FlpO) to remove the LacZ and Neo cassette, and generate a $t m 1 c$ allele (i.e. Cyp11a1 ${ }^{f l f l}$ ). When crossed with a Cre-driver, the Crerecombinase removes exon 3 of Cyp11a1 gene and creates a frameshift mutation (Fig. 1d). Because we had initially detected Cyp11a1 expression in Th2 cells ${ }^{28}$, we crossed the Cyp11alflfl line with a $C d 4$-driven Crerecombinase to delete Cyp11a1 and prevent de novo steroidogenesis in all $\mathrm{T}$ cells (Fig. 1e). Deletion efficiency of Crerecombinase in the Cyp11a1 cKO (Cd4-Cre;Cyp11a1 $\left.{ }^{f l f l}\right)$ mice was nearly complete in Th2 cells (Fig. 1f). Cyp11a1 cKO mice showed normal thymic development of $\mathrm{T}$ cells, and a normal distribution in the peripheral tissues (Fig. 1g-i).

In vitro analysis of Cyp11a1 expression in $\mathbf{T}$ cells. Exploiting our Cyp11a1-mCherry reporter line, we assayed a panel of cytokines commonly found in inflammatory settings, including tumors, for their ability to induce steroidogenesis in $\mathrm{CD}^{+}$ $\mathrm{T}$ cells. IL4, IL6, IL13, and TSLP induced a strong Cyp11a1mCherry signal in in vitro activated $\mathrm{CD} 4^{+} \mathrm{T}$ cells (Fig. 2a, Supplementary Fig. 2a, b). In contrast, IL12 had an inhibitory effect on Cyp11a1-mCherry expression (Fig. 2b, Supplementary Fig. 2c). This indicated that not only Th2 lymphocytes, but also other $\mathrm{T}$ cell types, are capable of de novo steroidogenesis, at least in vitro. To test this, we differentiated naïve $\mathrm{CD} 4^{+}$or $\mathrm{CD}^{+}$ T cells into Th1, Th2, Th9, Th17, Tfh, Treg, Tc1, and Tc2 subsets in vitro. All subsets examined, with the exception of Th1 and Tc1 cells, exhibited Cyp11a1-mCherry expression when activated, consistent with an inhibitory role for IL12 in Cyp11al regulation (Fig. 2c, Supplementary Fig. 2d). These data suggest that Cyp11a1 expression, and thereby steroidogenesis, is a default process during T cell activation, and is inhibited by the presence of IL12. Overall, of all $\mathrm{T}$ cell subtypes generated in vitro, Th2 cells exhibited the highest percentage of Cyp11a1 expression and Th1 cells do not express Cyp11a1.

To determine the requirement of Cyp11a1 activity for T helper cell proliferation and differentiation, we purified naïve splenic $\mathrm{T}$ cells from Cyp11a1 cKO and control mice. We activated the cells in vitro to generate different subclasses of T helper cells, and analyzed signature cytokine expression by flow cytometry. In the absence of Cyp11a1, T cells proliferate normally (Fig. 2d). Cyp11a1 expression was not required for the differentiation of any $\mathrm{T}$ helper cell type tested as determined by signature cytokine expression (Fig. 2e, f, Supplementary Fig. 2e). We observed that deletion of Cyp11a1 in $\mathrm{T}$ cells does not interfere with the plasticity of T helper cells (Fig. 2g, Supplementary Fig. 2f, g).

As a next step, Cyp11a1 induction in $\mathrm{T}$ cells was investigated in vivo.

Tumors induce functional Cyp11a1 expression in $\mathrm{T}$ cells. Tumor-infiltrating $\mathrm{T}$ cells are key fate determinants within a tumor, but are often suppressed ${ }^{30}$. The steroidogenesis-inducing type- 2 cytokines such as IL4 are also often present in the $\mathrm{TME}^{31,32}$, thus we next sought to examine the steroidogenic 
a

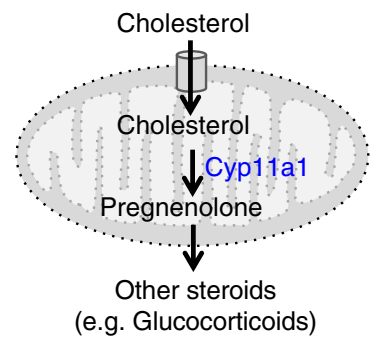

b

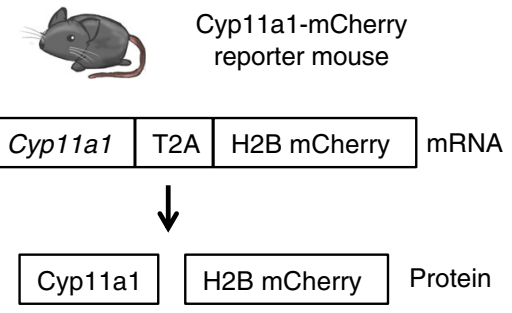

C

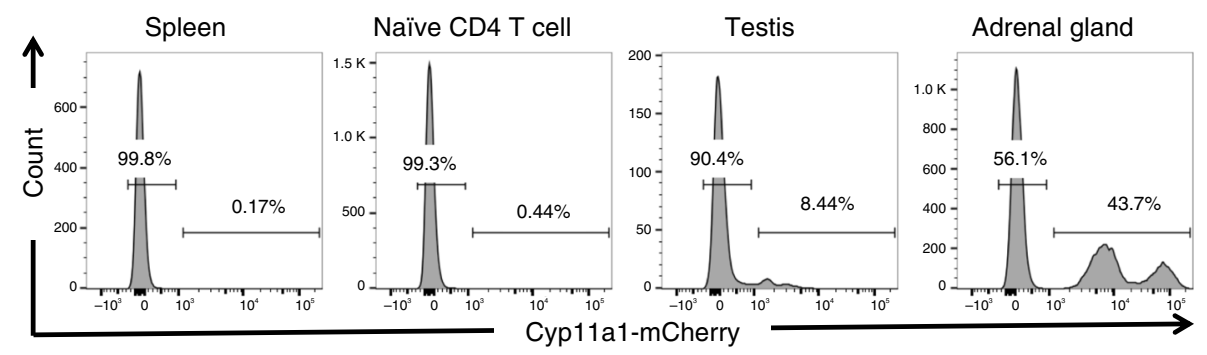

d

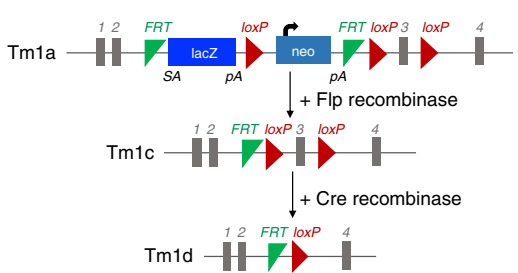

g

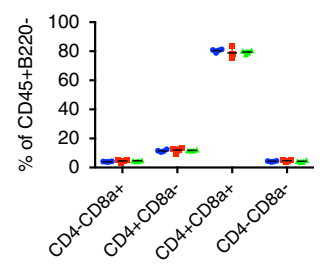

e

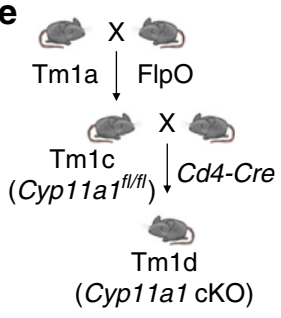

(Cyp11a1 cKO) $\mathbf{f}$

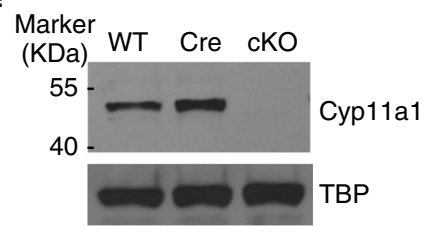

h

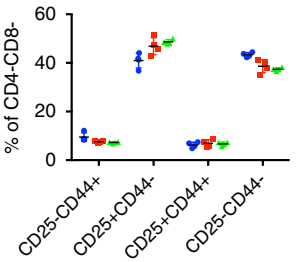

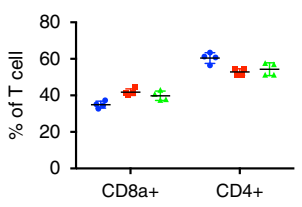

i

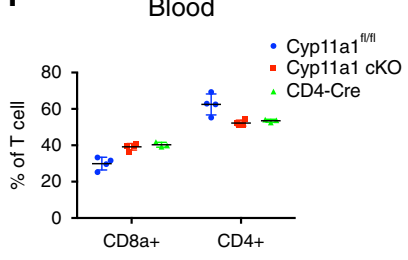

Fig. 1 Generation of a de novo steroidogenesis reporter and conditional knockout mice. a. Schematic of de novo steroidogenesis pathway. Cyp11a1 is the first and a key rate-limiting enzyme of the pathway. b. Cyp11a1-reporter mice synthesize a fusion protein that self-cleaves due to presence of a T2A peptide and dissociates into Cyp11a1 and H2B-mCherry. c Cyp11a1-mCherry reporter mice report Cyp11a1 expression accurately. Single-cell suspensions of tissues and naïve splenic CD4 + T cells were analyzed by flow cytometry. Gating: All cells $>$ Singlets $>$ Live cells $>C y p 11 a 1-m C h e r r y$. Representative of three independent experiments; each experiment contains 3-4 mice. d, e Generation of a Cyp11a1 conditional knockout (cKO) mice. Schematic presentation of the The targeting allele (d) and T cell-specific Cyp11a1 cKO (Cd4-Cre;Cyp11afl/fl) generation (e). $\mathbf{f}$ Cyp11a1 knockout efficiency of Cre recombinase in T cells. Splenic naïve T helper cells from cKO (Cd4-Cre;Cyp11a $7^{\mathrm{fl} / \mathrm{fl})}$ mice or control mice (wild type and Cd4-Cre) were activated under Th2 differentiation condition, and analyzed for Cyp11a1 protein expression by western blot. TATA-binding protein (TBP) used as loading control. $\mathbf{g}$ Normal thymic development of T cells in Cyp11a1 cKO. Thymus was harvested from Cyp11a1 cKO and control (Cd4-Cre and Cyp11a fl/fl) mice, dissociated into single-cell suspension, stained with fluorescent conjugated anti-CD4, CD8, B220, CD45, CD25, and CD44 antibodies, and analyzed by flow cytometry. Gating: all cells $>$ Singlets $>$ Live cells $>C D 45^{+}$B22 $20^{-}>C D 4, C D 8$ (left panel). CD $4^{+} \mathrm{CD} 8^{+}$cells represent double positive (DP) stage, $\mathrm{CD}^{+} \mathrm{CD} 8^{-}$and $\mathrm{CD} 44^{-} \mathrm{CD} 8^{+}$cells represent single positive (SP) stage, $C D 4^{-} \mathrm{CD} 8^{-}$cells represent double negative (DN) stage. DN cells of the left panel were gated to show $C D 25$ and CD44 expression to identify DN1 (CD25-CD44+), DN2 (CD25+CD44+), DN3 (CD25+CD44-), and DN4 (CD25-CD44-). Error bars represent mean with s.d., $N=4$ biologically independent animals. (h, i). Flow cytometric analysis shows normal distribution of Cyp11a1 cKO T cells (CD4 ${ }^{+}$and $\left.\mathrm{CD} 8^{+}\right)$in the peripheral blood and spleen. Error bars represent mean with s.d., $N=4$ biologically independent animals.

capacity (i.e. Cyp1la1 induction) of T cells infiltrating tumors, and their impact on tumor development. To explore Cyp11a1 expression in vivo, we utilized the well-established B16-F10 melanoma model ${ }^{33-35}$ and implanted tumors subcutaneously in Cyp11a1-mCherry reporter mice.

Cyp1la1 expression was detected in immune cells of established primary tumors, but not in tumor-draining brachial lymph nodes (LN) or blood (Fig. 3a), indicating that stimulation occurs in situ. In support of this, stimulation of splenic $\mathrm{T}$ cells did not induce Cyp11al expression ex vivo (Fig. 3b, Supplementary Fig. 3a). The dominant Cyp1la $1^{+}$tumor-infiltrating immune cells were identified as $\mathrm{T}$ cells, predominantly $\mathrm{CD} 4^{+}$(helper $\mathrm{T}$ cells, Fig. 3a).

Having identified presence of Cyp11a1-expressing $\mathrm{T}$ cells in established tumors, we next examined expression dynamics during tumor progression. Using the Cyp11a1-mCherry reporter 


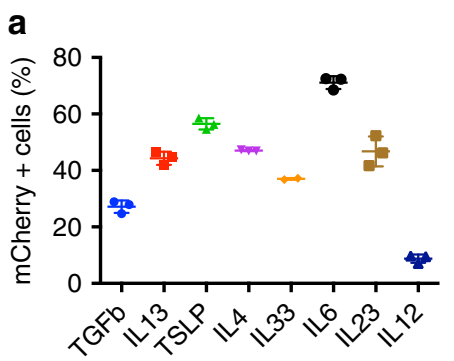

b

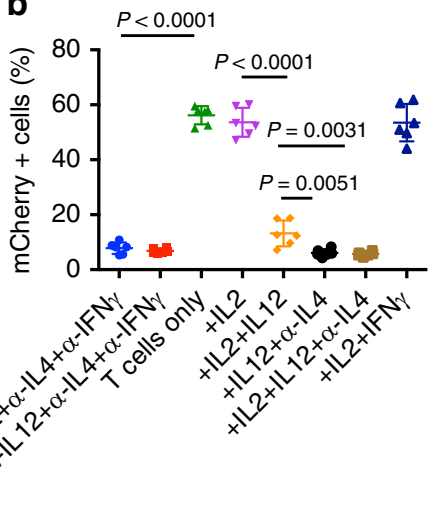

C

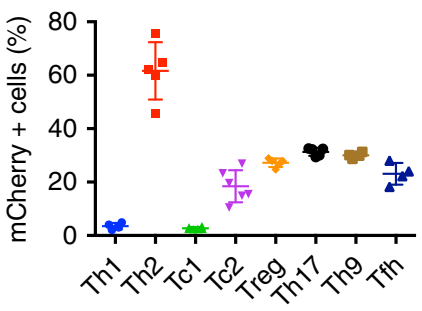

\section{$x^{+a^{2}}$}

d
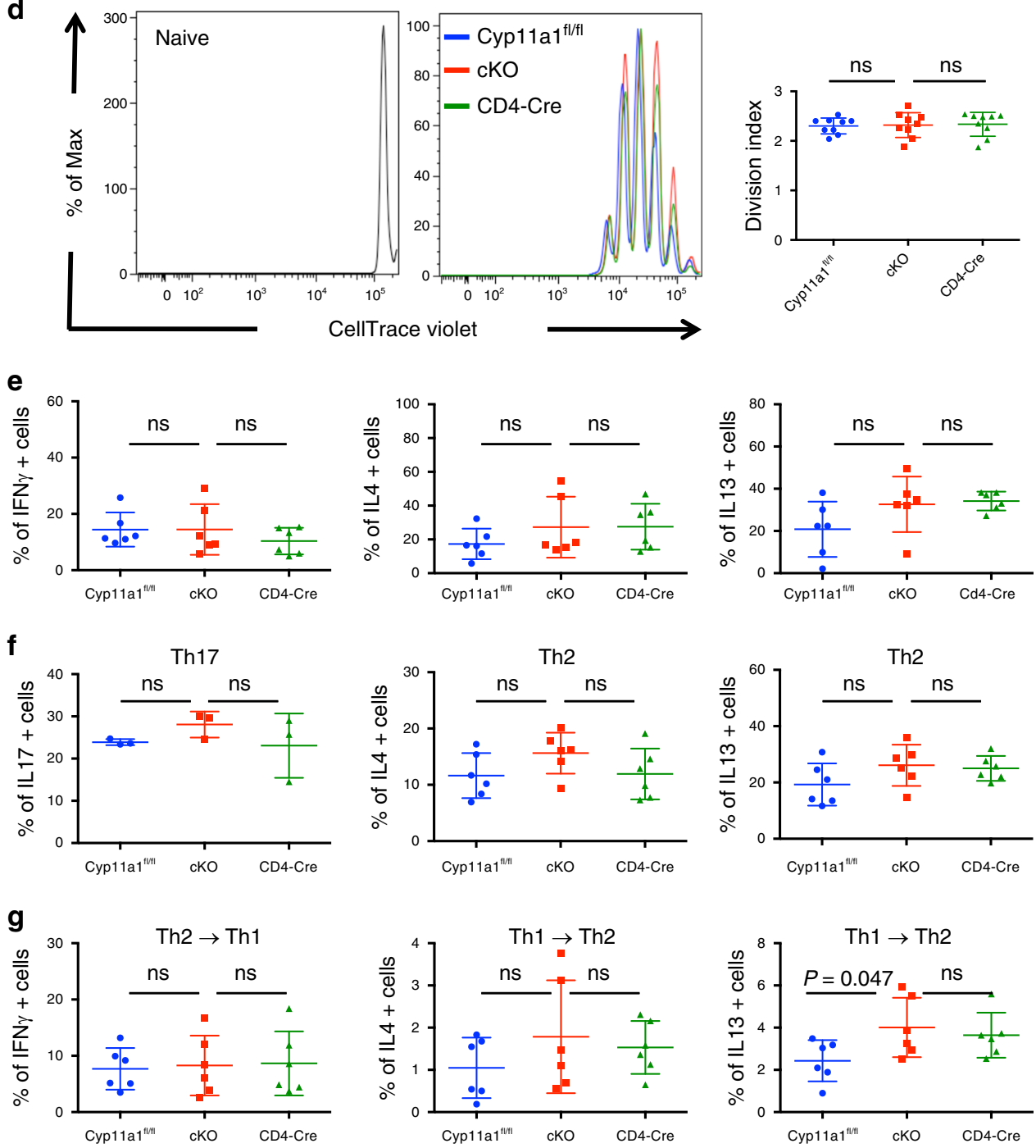

mice we observed expression of Cyp11a1 in $\mathrm{CD} 4^{+} \mathrm{T}$ cells which remained stable during development of the tumor through days 7 and 12. By contrast, $\mathrm{CD} 8^{+} \mathrm{T}$ cells only expressed Cyp11a1 by day 12 (Fig. 3c, d). Expression dynamics of minor and rare Cyp11a1 ${ }^{+}$ non-T cells (mast cells and basophils) is shown in Supplementary Fig. 3b, c.

To determine whether Cyp1lal induction in $\mathrm{T}$ cells is conserved in other tumor types we analyzed an EO771 orthotopic model of breast cancer ${ }^{36-38}$ (Fig. 3e-g, Supplementary Fig. 3d). Again, we found that Cyp1la1 was upregulated in tumor- infiltrating T cells (Fig. 3e-g), but not in tumor-draining lymph node or spleen. We also detected minor and rare populations of other Cyp1la $1^{+}$type- 2 immune cells, such as mast cells and basophils (Supplementary Fig. 3d).

Next, we sought to measure the functional output of Cyp1la1 expression. Significant concentrations of the steroid pregnenolone were detected exclusively in immune cells isolated from tumors, with negligible levels detected in cells from the spleen (Fig. 3h). Using the B16-F10 model of experimental metastatic dissemination $^{39}$, we determined that lungs with metastatic nodules, but not 
Fig. 2 Analysis of Cyp11a1-expressing T cells using Cyp11a1 reporter and cKO mice. a Splenic naïve CD4+ T cells from Cyp11a1-mCherry reporter mice were purified by negative selection; activated in the anti-CD3e/anti-CD28 antibody coated plates in the presence of cytokines for 3 days, rested for 2 days, restimulated $6 \mathrm{~h}$, and Cyp11a1-mCherry expression was analyzed by flow cytometry. $N=3$ (except IL33 where $N=2$ ). b IL12 inhibits Cyp11a1 expression. Splenic naïve CD4+ T cells from Cyp11a1-mCherry reporter mice were activated as mentioned above (a) for 5 days and Cyp11a1-mCherry expression was analyzed by flow cytometry. $N=6$. c Splenic naïve $C D 4^{+}$and $C D 8^{+}$T cells from Cyp11a1-mCherry reporter mice were activated in vitro under Th1, Th2, Th9, Th17, Tfh, Treg, Tc1, and Tc2 differentiation conditions (activation 3 days, resting 2 days), and Cyp11a1-mCherry expression was analyzed by flow cytometry. $N=6(T c 1, T c 2), N=4$ (Th1, Th9, Tfh), and $N=5$ (Treg, Th17). d Splenic naïve CD4+ T cells were purified from Cyp11a1 cKO, Cd4-Cre, and Cyp11a $7^{\mathrm{fl} / \mathrm{fl}}$ mice, stained with CellTrace Violet, activated in vitro, and cell proliferation was determined by a flow cytometric dye decay assay.

Representative cell proliferation profiles are shown in the left panel and a comparison of the cell division index is shown in right panel. $N=9$. e Splenic naïve $\mathrm{CD} 4{ }^{+} \mathrm{T}$ cells were activated in vitro in the absence of any exogenous cytokine or cytokine-neutralizing antibody, and cytokine expression was determined by flow cytometry. $N=6$. f Splenic naïve $C D 4^{+} \mathrm{T}$ cells were activated in vitro under Th17 or Th2 differentiation conditions, and cell typespecific signature cytokine expression was determined by flow cytometry. $N=3$ (Th17), $N=6$ (Th2). $\mathbf{g}$ Splenic naïve CD4 ${ }^{+} T$ cells were activated in vitro under Th1 or Th2 differentiation condition. After 3 days Th1 cells were allowed to differentiate under Th2-polarizing conditions (Th1 > Th2), and Th2 cells were allowed to differentiate under Th1-polarizing conditions (Th2 > Th1). Th1 or Th2-specific cytokine expression was determined by flow cytometry. $N=$ 6. All error bars in this figure represent mean with s.d. $P$-value was calculated using unpaired two-tailed $t$-test. Representative of three independent experiments. $N$ represents biologically independent animals.

control lungs without metastatic nodules, had elevated levels of pregnenolone (Fig. 3i).

The presence of tumor cells did not induce or augment the Cyp1lal expression in $\mathrm{T}$ cells in vitro (Supplementary Fig. 3e). This indicates that type-2 (Th2) cytokines within the TME induce Cyp1la1 expression by $\mathrm{T}$ cells and this cannot readily be mimicked in vitro.

Having observed steroidogenic T cells in murine melanoma, we turned to publicly available transcriptomic data sets to verify our findings and ascertain relevance in the human setting. We identified CYP11A1 mRNA expression, and thus steroidogenic potential, in a range of cancer types including liver, breast, prostate, lung, kidney, sarcoma, glioma, uterine, cervical, lymphoma, and melanoma (Supplementary Fig. 3f, g) ${ }^{32}$. Human melanoma tissues represented a prominent steroidogenic tumor type, expressing CYP11A1, HSD3B1, HSD3B2, CYP17A1, $C Y P 21 A 1$, and $C Y P 11 B 1$ and not expressing CYP11B2 (Fig. 3j, $\mathrm{k})$. CYP11B2 catalyzes the aldosterone synthesis from corticosteroid precursor (Fig. 3k). Together this was indicative of melanoma-driven production of glucocorticoids (Fig. 3k, Supplementary Fig. 3h).

Interestingly, in melanoma, steroidogenic gene expression was correlated with IL4 expression (Fig. 3k, Supplementary Fig. 3j), a key inducer of $\mathrm{T}$ cell steroidogenesis ${ }^{28}$. Moreover, analysis of human tumor-infiltrating CD4 ${ }^{+} \mathrm{T}$ cell transcriptomes, confirmed CYP11A1 expression (Fig. 31) implying that CD4 ${ }^{+} \mathrm{T}$ cells are a source of steroids in tumors, mirroring the murine setting. Collectively these data indicate that in both human and mice, TILs produce steroids within the tumor.

Gene expression identity of Cyp11a1 ${ }^{+}$immunocytes in melanoma. To reveal the gene expression identity of intratumoral steroidogenic immune cells and patterns of gene expression at single-cell resolution, we inoculated B16-F10 subcutaneous tumor in Cyp11a1-mCherry reporter mice, enriched and purified intratumoral Cyp11a1-mCherry ${ }^{+}$and Cyp11a1-mCherry ${ }^{-}$ cells by cell sorting into 96-well plates [with a ratio of 79:15 $\left(\mathrm{mCherry}^{+}:\right.$mCherry $^{-}$) cells per plate] and performed single-cell RNA sequencing (scRNA-seq) using the SMART-Seq2 platform ${ }^{40}$. The majority of cells, $\sim 3000$, passed quality control. Unsupervised hierarchical clustering and visualization with reduced dimensionality using UMAP (uniform manifold approximation and projection $)^{41}$ identified 12 clusters of cells (Supplementary Fig. 4a). There was no batch effect between samples (Supplementary Fig. 4b) or impact of sex (Supplementary Fig. 4c) on clustering, and Cyp11a1-mCherry protein expression accurately reported Cyp11a1 mRNA expression (Supplementary Fig. $4 \mathrm{~d}, \mathrm{e})$. We annotated the clusters based on classical lineage markers (Supplementary Fig. 4a, f) and their signature gene expression (Supplementary Fig. 4g). The majority of the Cyp11a1-expressing cells are $\mathrm{T}$ cells as suggested by $\mathrm{Cd} 3 e$ expression (Supplementary Fig. 4f). T cells constitute the clusters $0,1,2,3$, and 8 with cluster- 1 displaying genes of CD8 ${ }^{+} \mathrm{Tc} 2$ cells; Cd8a, Cd3e, Gata3, and type 2 cytokines Il4, Il5, and Il13. Clusters 0,2 , and 3 were $\mathrm{CD} 4^{+} \mathrm{T}$ helper cells $\left(\mathrm{Cd} 4^{+} \mathrm{Cd} 3 e^{+}\right)$as they expressed Gata3, Il4, and Il13 (Supplementary Fig. 4f). As $\mathrm{T}$ cells were the dominant Cyp11a1-expressing population, we reanalyzed the data taking only the $\mathrm{T}$ cells and displayed in Fig. $4 \mathrm{a}-\mathrm{e}$. The three clusters of Th2 cells represent three closely related subpopulations (Fig. 4a, b, Supplementary Fig. 4a). The comparative gene expression between these clusters is shown in Supplementary Fig. 4i. In Supplementary Fig. 4a, Cluster-4 represents myeloid derived cells, macrophages, and dendritic cells, but mostly they constitute Cyp11a1-mCherry ${ }^{-}$cells, though a very small number of Cyp11a1-mCherry ${ }^{+}$cells have been found in this cluster. Cluster-5 contains B16-F10 cells and melanocytes from the host mice, and they were Cyp11a1 ${ }^{-}$. Cluster- 6 and 9 constitute two different states of the Cyp11a1 ${ }^{+}$mast cells, because they express Kit (the gene that encodes cKit) and Fcerl and other mast-cell-specific proteases such as Mcpt4 and Cmal. The detailed differences between these two clusters have been shown in Supplementary Fig. 4j. Cyp11a1 ${ }^{+}$basophils and eosinophils (cluster-7) fall in the same cluster. Cluster-8 is a mixed population of $\mathrm{CD}^{+}{ }^{+}$and $\mathrm{CD} 8^{+} \mathrm{T}$ cells. The number of cells in these two clusters are not sufficient to make a conclusion of their identity. Clusters 4, 5, 8, 11, and 10 are constituted by both Cyp11a1mCherry $^{+}$and Cyp11a1-mCherry ${ }^{-}$cells. Cyp11a1-mCherry ${ }^{-}$ cells representing the cells that are most frequently available cells within B16-F10 tumor, namely B16-F10 cells and macrophages.

Taking Cyp11a1-correlated genes we ran pySCENIC to identify potential transcription factors regulating Cyp 11a1 expression and found that the GATA family of transcription factors (Gata1, 2, 3) were predicted to be the controller of the Cyp11a1 locus in these steroidogenic immune cells (Supplementary Fig. 4h). Gata3 is the lineage-defining TF of Th2 cells. To validate the prediction that Gata3 controls Cyp11a1 expression in $\mathrm{T}$ helper cells, first we performed ATAC-seq to identify the open chromatin and potential locus control region (Fig. 4f). Next, we analyzed Gata3 ChIP-Seq data to confirm that Gata3 is indeed a potential regulator of the Cyp11a1 locus in Th2 cells (Fig. 4f). The putative locus control region (blue highlighted region in Fig. 4f) was found in a closed-chromatin state in naïve and Th1 cells but was open in 
a

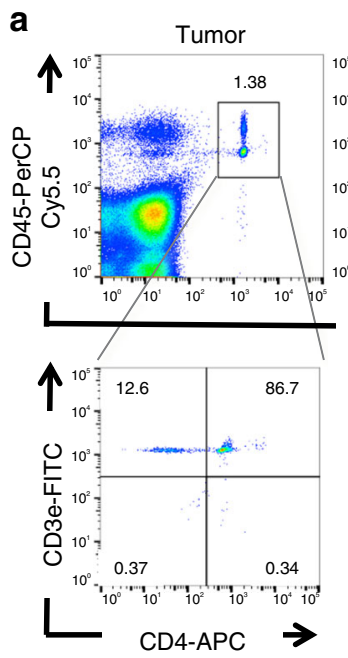

Blood

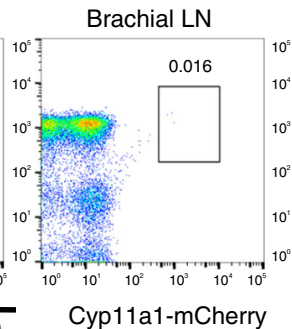

Cyp11a1-mCherry

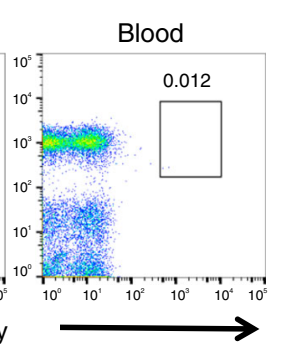

b

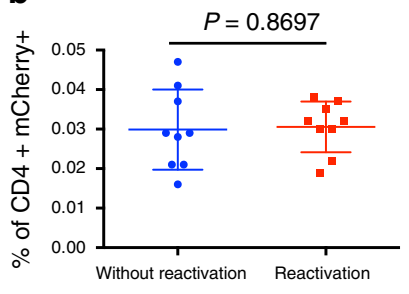

c
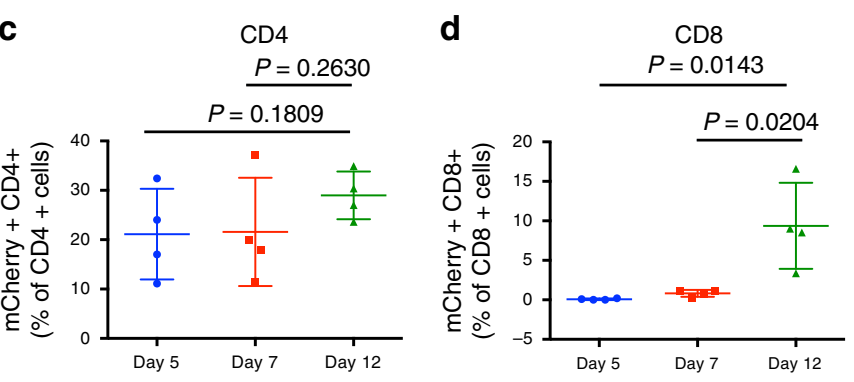

e
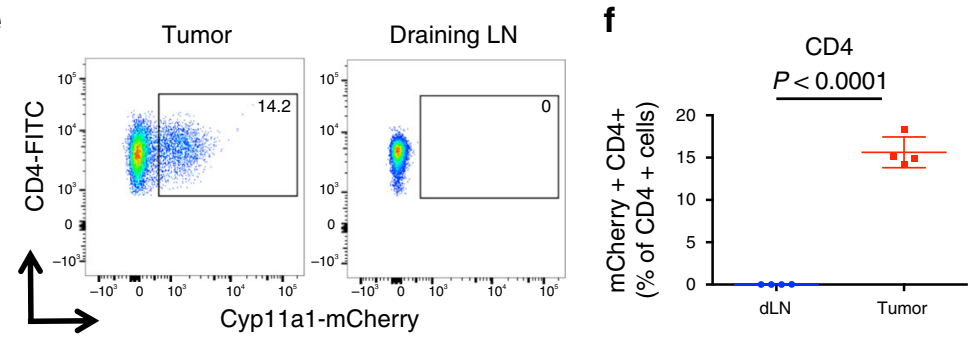

g
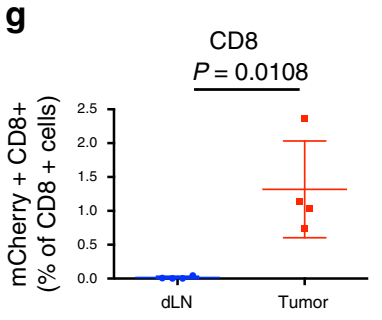

h

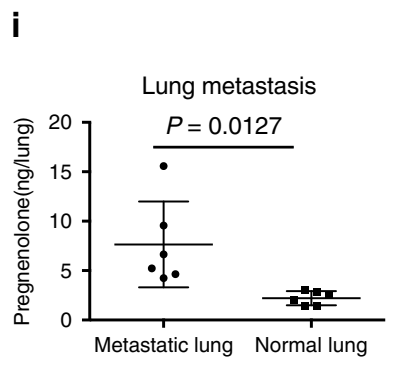

j

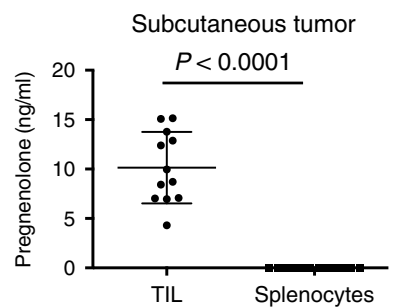

k
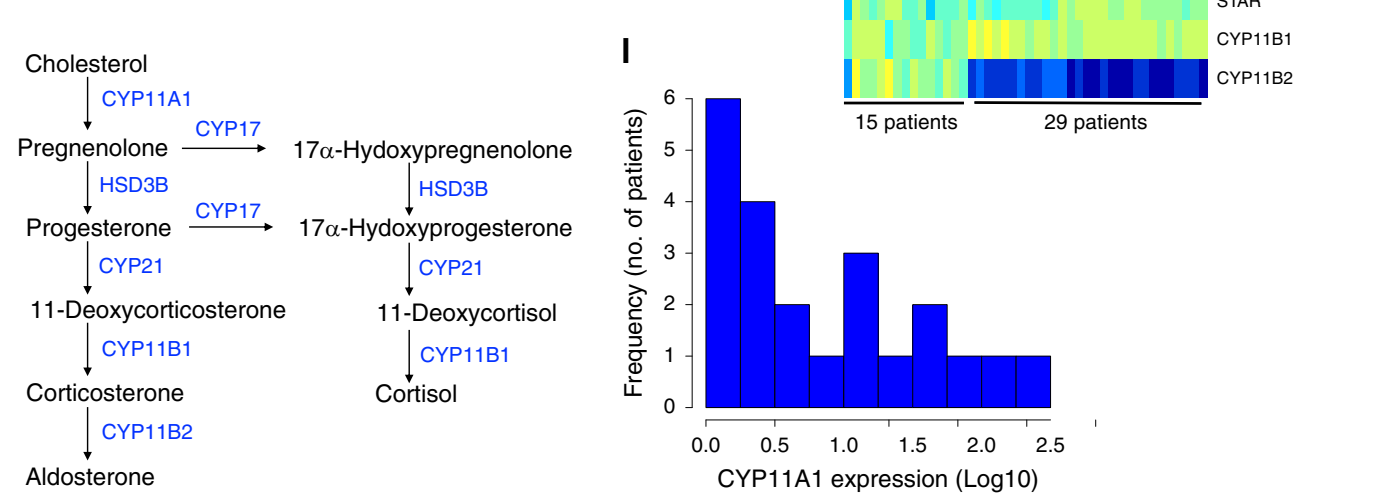

Th2 and Th17 cells and was occupied by Gata3 in Th2 cells (Fig. 4f).

All the Cyp11a1-expressing T cells express steroidogenic type-2 cytokines Il4 and Ill3 (Fig. 4e, Supplementary Fig. 4f). T cells express the cognate receptor for IL4 (Il4ra) indicating that these cells are receptive to the steroidogenic signal (Supplementary Fig. 4f).

The prediction of glucocorticoids in the tumor prompted us to check the cognate receptors in the tumor-infiltrating immune

cells. We found that the glucocorticoid receptor $\mathrm{Nr} 3 \mathrm{cl}$ was expressed in all tumor-infiltrating immune cells (Supplementary Fig. 4k).

Ablation of $\mathrm{T}$ cell steroidogenesis restricts tumor growth. To determine the functional consequences of $\mathrm{T}$ cell-driven steroidogenesis in tumors we subcutaneously implanted Cyp11a1 cKO mice with B16-F10 cells. Genetic deletion of Cyp11a1 in 
Fig. 3 Tumors induce Cyp11a1 expression in T cells in vivo. a B16-F10 cells were injected subcutaneously into the Cyp11a1-reporter mice. After 12 days brachial lymph node (LN), blood, and tumor tissues were analyzed by flow cytometry. Gating strategy: Singlets $>$ Live cells $>C D 45, C y p 11 a 1-m C h e r r y . ~ N=5$. CD45 ${ }^{+}$Cyp11a1-mCherry ${ }^{+}$cells were further gated to show T helper cell $\left(C D 4+C D 3 e^{+}\right)$expression of Cyp11a1. b Splenic cells were purified from tumorbearing Cyp11a1-reporter mice, and restimulated in vitro using PMA/ionomycin and analyzed by flow cytometry. N=9. c, d B16-F10 cells were injected subcutaneously into the Cyp11a1-mCherry reporter mice. After 5, 7, and 12 days tumor tissues were analyzed by flow cytometry to detect the CD4+ ${ }^{+}$cells (c), $\mathrm{CD} 8{ }^{+} \mathrm{T}$ cells $(\mathbf{d}) \mathrm{N}=4$. All cell $>$ Singlets $>$ Live cell $>\mathrm{CD} 45^{+}>\mathrm{CD} 4^{+} \mathrm{CD} 3 \mathrm{e}^{+}$or $\mathrm{CD} 8^{+} \mathrm{CD} 3 \mathrm{e}^{+}$. e-g EO771 cells were injected into the mammary fat pad of Cyp11a1-reporter mice. After 15 days, tumor tissues and tumor-draining LN were analyzed by flow cytometry to detect the Cyp11a1-mCherry expression in CD4 ${ }^{+} \mathrm{T}$ cells $(\mathbf{e}, \mathbf{f}) \mathrm{CD}^{+} \mathrm{T}$ cells $(\mathbf{g})$. Gating: All cell>Singlets $>$ Live cell>CD45 ${ }^{+}>\mathrm{CD} 4{ }^{+} \mathrm{TCRb}{ }^{+}$or $\mathrm{CD} 8^{+} \mathrm{TCRb}{ }^{+}$. e Representative FACS profile of Cyp11a1 ${ }^{+}$ CD4+ T cells. $\mathbf{f}$, $\mathbf{g}$ Representative graphical presentation of one experiment showing Cyp11a1-expressing CD4 and CD8 T cells. N=4. $\mathbf{h}$ B16-F10 tumorinfiltrating leukocytes (TIL) and splenocytes were purified from tumor-bearing mice on post-inoculation day-12, cultured for $48 \mathrm{~h}$, and the supernatant was analyzed by ELISA to measure pregnenolone. $N=12$, pooled analysis of three independent experiments. $\mathbf{i}$ Metastasized lungs were harvested 10 days post-B16-F10 intravenous injection in C57BL/6 mice, dissociated into single-cell suspension, cultured for 48 h, and the supernatant was analyzed by ELISA. Naïve uninfected lungs (normal lung) were used as control. $N=6$, pooled analysis of two independent experiments. $\mathbf{j}$ Hierarchical clustering of steroidogenic genes and IL4 mRNA expression across 44 melanoma patient samples (GEO: GSE19234). k Schematics showing glucocorticoid synthesis pathway. I Frequency distribution histogram showing CYP11A1 mRNA expression (normalized read counts, log10 scale) across 22 melanoma patients' tumor-infiltrating CD4+ T cells (EGAD00001000325). Individual data points for CYP11A1 expression are shown in Supplementary Fig. 3i. All error bars in this figure represent mean with s.d. P-values were calculated by unpaired two-tailed $t$-test. $N$ represents biologically independent animals.

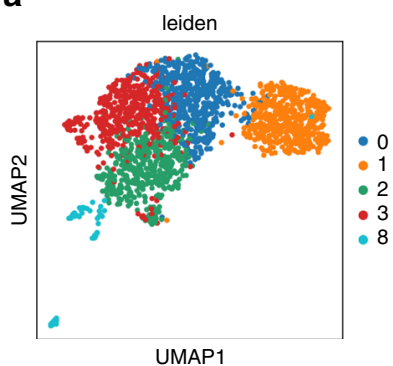

e

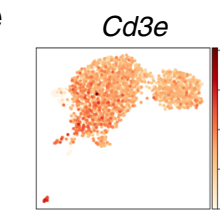

Tbx21

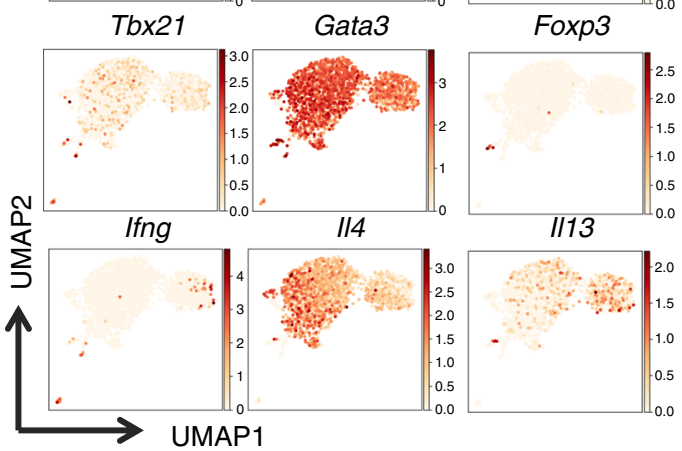

b Annotation

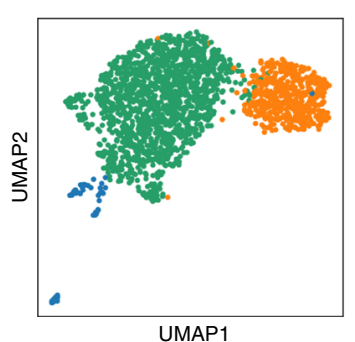

UMAP1
C

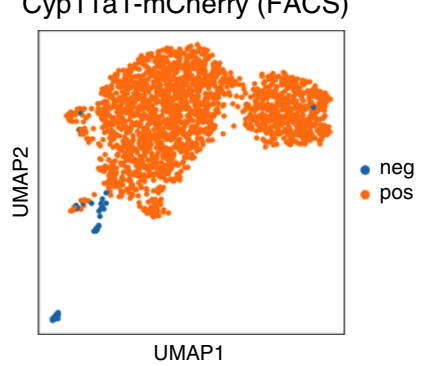

d Cyp11a1 (scRNAseq)

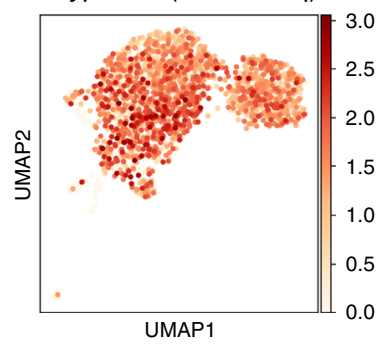

f

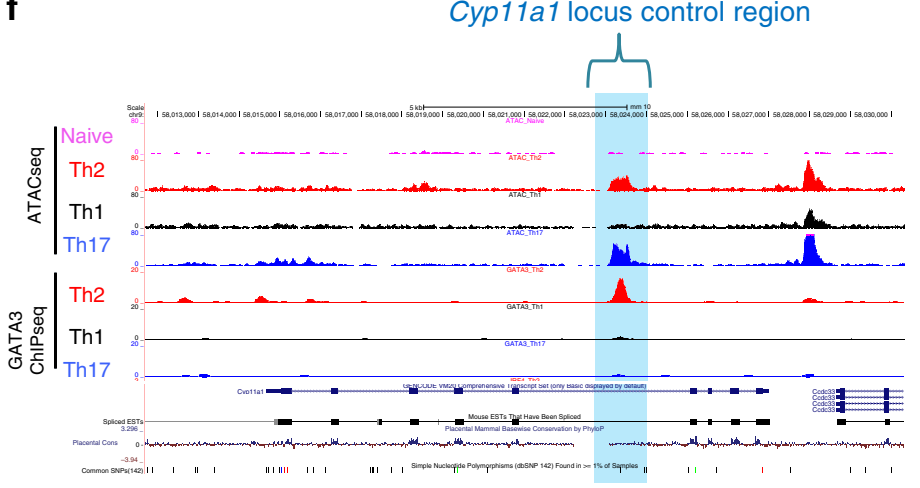

Fig. 4 Revealing gene expression identity of intratumoral Cyp11a1 ${ }^{+} \mathbf{T}$ cells by scRNAseq. $\mathbf{a}, \mathbf{b}$ UMAP visualization of the tumor-infiltrating $T$ cells (a) with annotations of the clusters (b). Tc1 represents type-1 CD8 ${ }^{+} \mathrm{T}$ cells, Tc2 represents type- 2 CD8 ${ }^{+} \mathrm{T}$ cells, and Th2 represents type-2 CD4 ${ }^{+} \mathrm{T}$ cells. c, $\mathbf{d}$ mCherry protein expression accurately reports Cyp11a1 mRNA expression. Cyp11a1-mCherry protein expression according to the flow cytometry (FACS) data (c). Cyp11a1 mRNA expression in the scRNA-seq data (d). e Expression of cell-type-specific signature genes that were used to annotate the clusters. $\mathbf{f}$ Determination of Cyp11a1 locus control region. Open chromatin regions were identified by ATAC-seq of T helper cells. In vitro generated Th2 and Th17 cells were used as positive control. Naïve and Th1 cells were used as negative control. Gata3 ChIP-seq data were analyzed to determine the binding site. The open chromatin region where Gata3 occupy is highlighted blue and considered as locus control region.

$\mathrm{T}$ cells significantly restricted primary tumor growth rates and final volumes (Fig. 5a). Similarly, in the experimental metastasis model, impaired lung colonization was observed in the absence of $\mathrm{T}$ cell-expressed Cyp11a1. There was a significant reduction in number of metastatic foci in lungs of Cyp11a1 cKO mice compared to the control mice (Fig. 5b). In the B16-F10 subcutaneous melanoma model, topical application of exogenous pregnenolone at the primary tumor site was sufficient to compensate for the Cyp11a1 deficiency, restoring tumor growth to levels comparable with control mice (Fig. $5 \mathrm{c}$ ). This suggests that the tumor restriction was a consequence of the absence of pregnenolone synthesis. Similar results were obtained using the EO771 breast cancer orthotopic tumor model: deletion of Cyp11a1 in $\mathrm{T}$ cells restricted dissemination to and nodule 
a
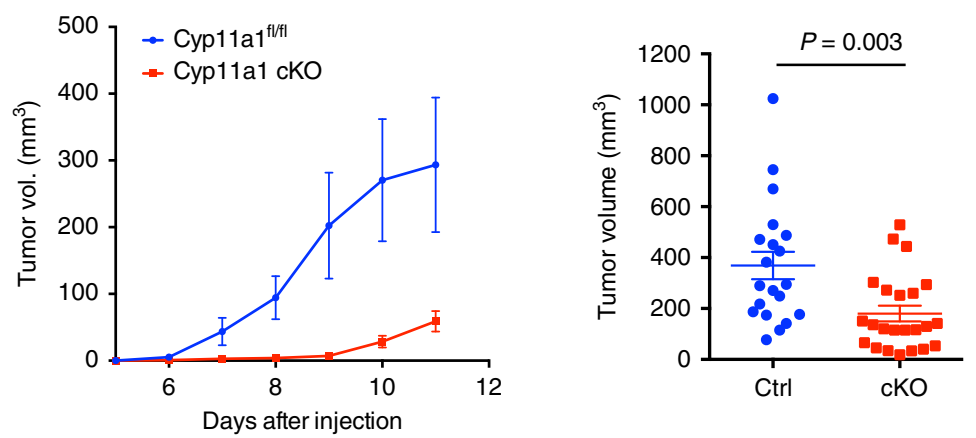

b
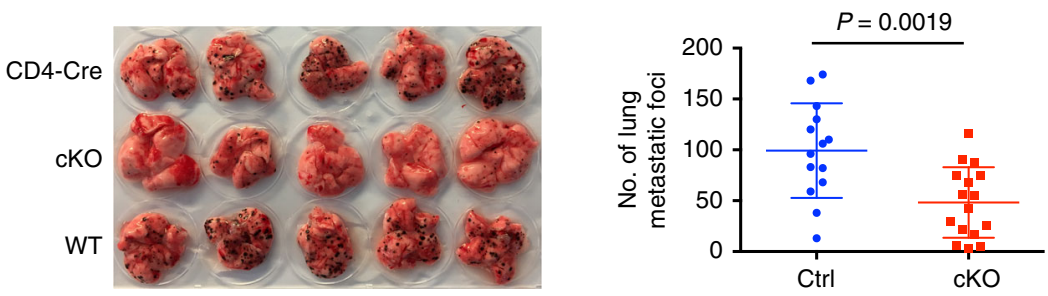

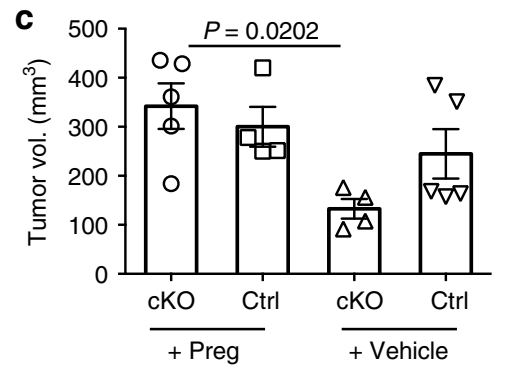

e

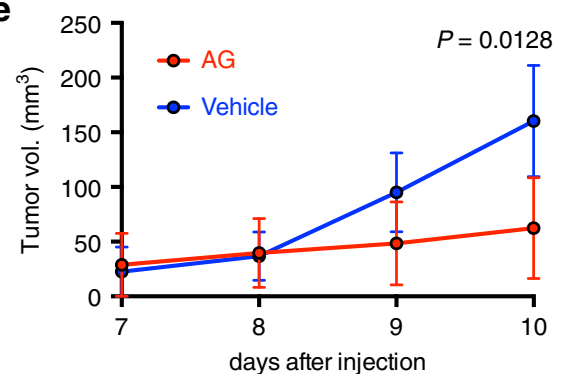

d
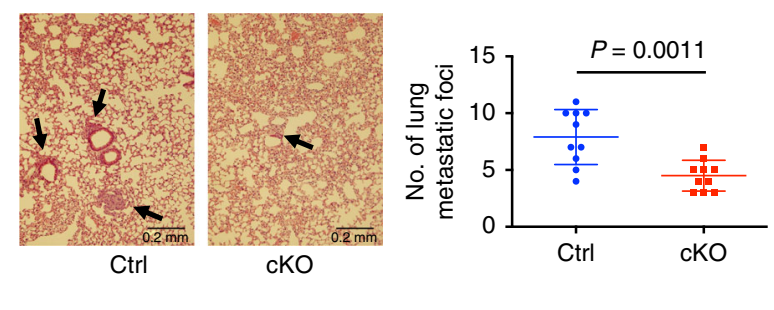

$\mathbf{f}$

Fig. 5 Ablation of $\mathbf{T}$ cell steroidogenesis restricts experimental tumor growth and metastasis. a Left-panel: B16-F10 subcutaneous tumor growth curve assessed in T cell-specific Cyp11a1 cKO (cKO) and Cd4-Cre control mice. $N=5$. Representative of four independent experiments. Right panel: graphical presentation of end-point tumor volume. $N=20$ (ctrl), $23(\mathrm{cKO})$. Pooled data of four independent experiments. Each point represents an individual animal. Error bars represent mean \pm s.e.m., unpaired two-tailed $t$-test. b Left panel: Representative photograph of pulmonary metastatic foci produced 10 days after tail vein injection of B16-F10 cells in CKO and control (Cre and WT) mice. $N=5$. Right panel: graphical presentation of the numbers of lung metastatic foci. $N=14$ (ctrl), 16 (cKO), pooled data of three independent experiments, each point represents an individual animal, error bars represent mean with s.d. Unpaired two-tailed t-test. c cKO and Cd4-Cre control mice were injected with B16-F10 cells. Pregnenolone or vehicle (DMSO) applied topically at the primary tumor site every $48 \mathrm{~h}$. Tumor volume was measured at the end-point at day $12 . N=5$. Each point represents an individual animal. Error bars represent mean \pm s.e.m. P-value was calculated by one-way ANOVA with Tukey Post hoc test. $\mathbf{d}$ Representative hematoxylin and eosin stained histologic photograph of pulmonary metastatic foci produced 10 days after tail vein injection of EO771 cells in Cyp11a1 cKO and Cyp11a $7^{\mathrm{fl} / \mathrm{fl}}$ mice. Graphical presentation of the numbers of lung metastatic foci (right panel). $N=10$. Error bars represents mean with s.d. Unpaired two-tailed $t$-test. Representative of two independent experiments. e B16-F10 cells were injected subcutaneously in C57BL/6 mice with or without Cyp11a1 inhibitor aminoglutethimide (AG). AG treatment was continued with a $48-h$ interval. $N=5$. Error bars represent mean \pm s.e.m. Two-way ANOVA. Representative of two independent experiments. $\mathbf{f}$ EO771 cells were injected into the mammary fat pad in C57BL/6 mice with or without Cyp11a1 inhibitor AG. AG treatment was continued with a 48 -h interval. $N=5$. Error bars represent mean \pm s.e.m. One experiment. In this figure, $N$ represents biologically independent animals.

formation in the lung (Fig. 5d). Importantly, in both tumor models, pharmacological inhibition of Cyp11a1 by aminoglutethimide (AG) was sufficient to recapitulate the tumor restriction phenotype resulting from Cyp11a1 genetic ablation
(Fig. 5e, f). Together, these data indicate that T-cell-derived steroids can support tumor growth and both genetic and pharmacological interference with the pathway can restrict tumor growth. 
a

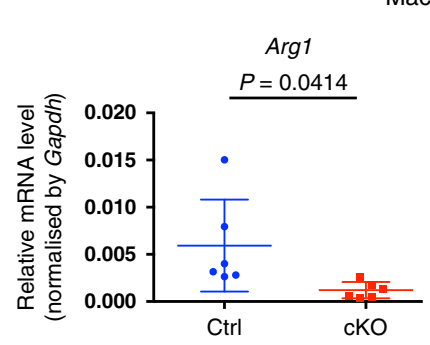

Macrophage
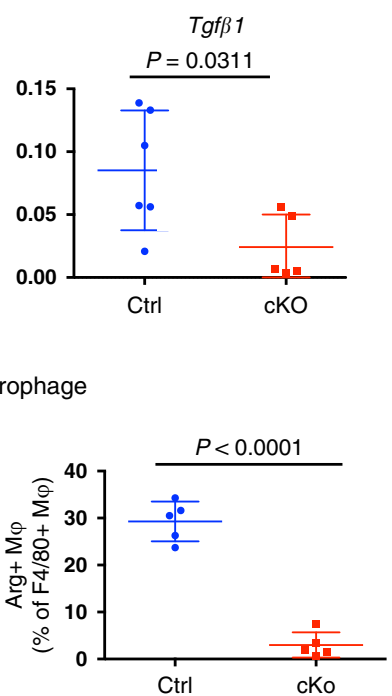

e
Macrophage

f

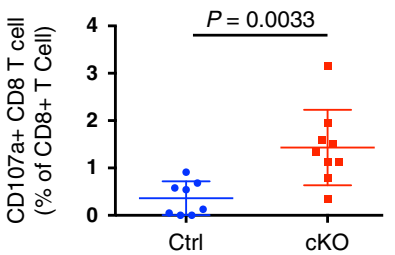

C
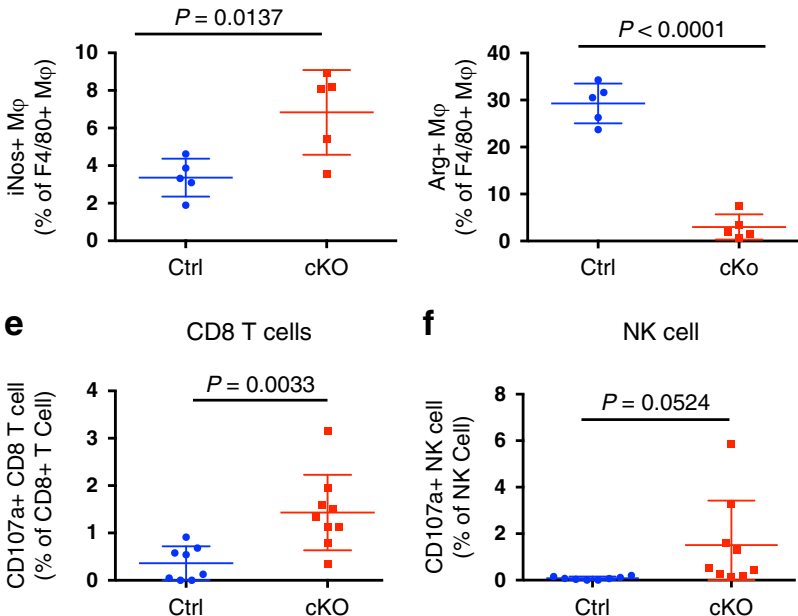

b

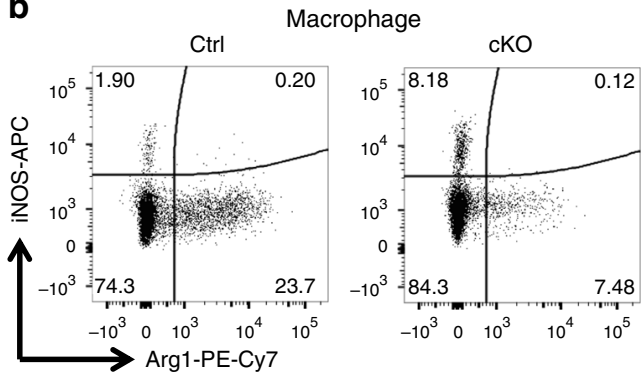

d

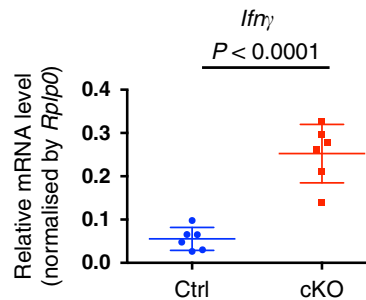

CD8 T cells

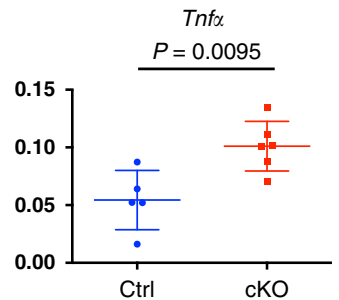

g

CD4 T cells

h

Treg
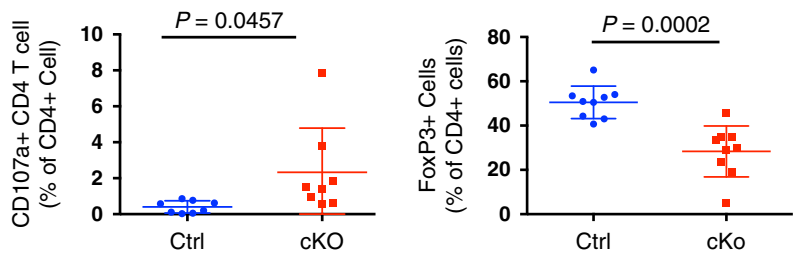

i

Macrophage
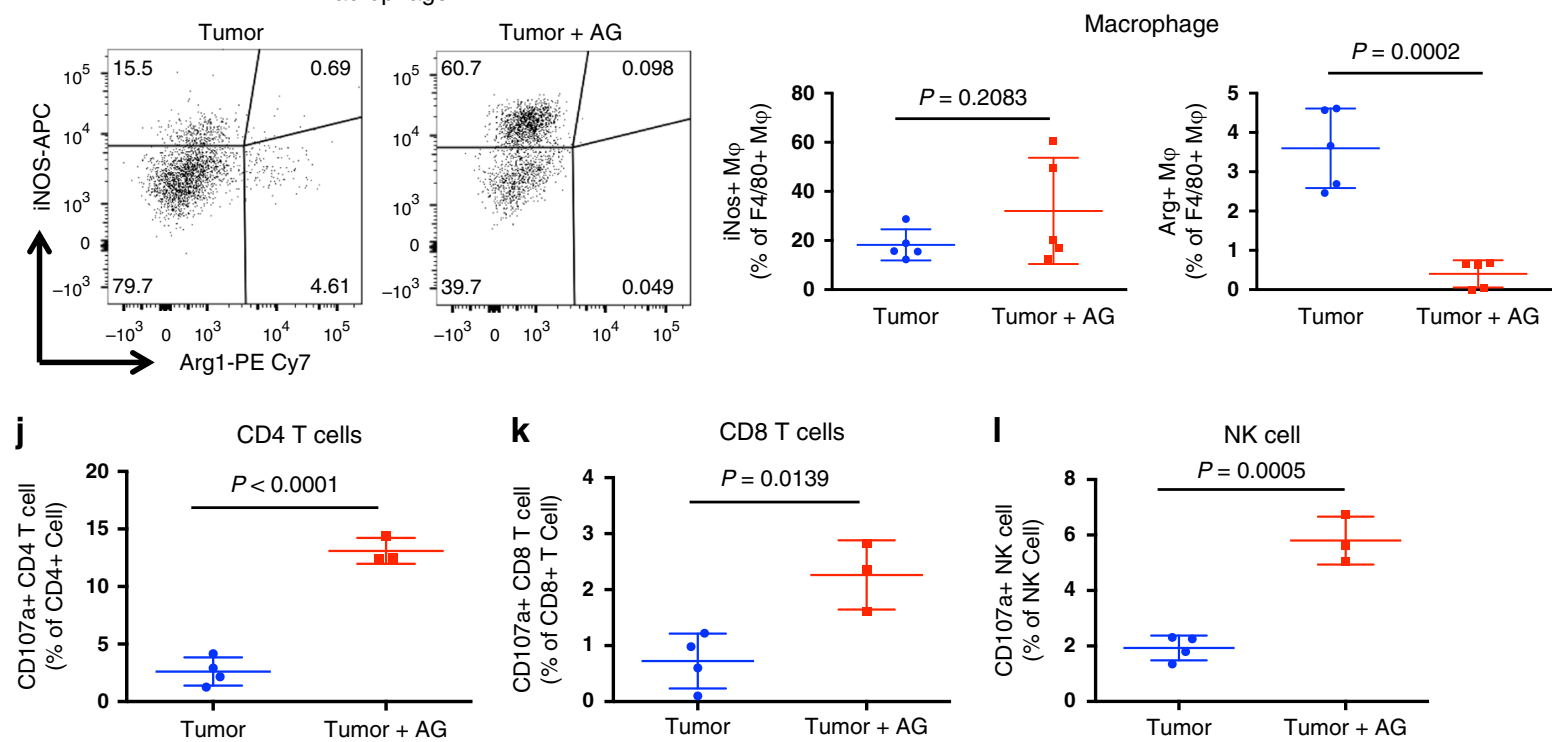

Inhibition of $\mathrm{T}$ cell steroidogenesis boosts tumor immunity. Steroid hormones can induce an immunosuppressive M2-like phenotype in macrophages ${ }^{26,42,43}$ cell death and anergy in $\mathrm{T}$ cells ${ }^{26,44-46}$, tolerance in dendritic cells ${ }^{26,47-49}$ and increase the frequency of Treg cells ${ }^{50-52}$. Therefore, we tested whether steroidogenic $\mathrm{T}$ cells support tumor growth through the induction of immunosuppressive phenotypes in infiltrating immune cells. To determine whether intratumoral macrophages were M1 or M2 type, we purified tumor-infiltrating macrophages and analyzed mRNA expression of the M2-macrophage signature genes Arg1 and Tgf $\beta 1$. In tumor-infiltrating macrophages from Cyp 11a1 cKO mice, Arg1 and $\operatorname{Tg} f \beta 1$ mRNA expression was significantly reduced compared to the control mice, indicating fewer of the tumorsupporting M2 macrophages in the $\mathrm{T}$ cell-specific Cyp11a1 knockout mice (Fig. 6a). We confirmed changes in macrophage characteristics at the protein level, showing a significant decrease in $\mathrm{Arg} 1^{+}$macrophages (M2) with coincident increase in tumorinfiltrating iNOS $^{+}$macrophages (M1) upon $\mathrm{T}$ cell-specific Cyp11a1 deletion (Fig. 6b, c). Hence, depletion of Cyp11a1 in $\mathrm{T}$ cells increases the M1/M2 macrophage ratio in the TME.

Consistent with enhanced tumor immunity, we found significantly higher levels of the inflammatory cytokines Ifn $\gamma$ and $T$ nf $\alpha$ expression in $\mathrm{CD}^{+} \mathrm{T}$ cells following Cyp11a1 ablation (Fig. 6d). Moreover the expression of the co-inhibitory 
Fig. 6 Inhibition of T cell steroidogenesis stimulates anti-tumor immunity. a-h Comparing Cyp11a1 cKO and Cd4-Cre control mice with B16-F10 cells injected subcutaneously. Representative of three independent experiments. a Tumor-infiltrating macrophages (Lin ${ }^{-} \mathrm{CD}_{11 b}{ }^{+}$) were purified by cell sorting at day 12. Arg1 and TgfB1 mRNA expression was quantified by RT-qPCR, with mRNA expression level normalized by Gapdh mRNA expression. $N=6$.

b, c Tumor-infiltrating macrophages ( $\mathrm{Lin}^{-} \mathrm{CD} 11 \mathrm{~b}^{+} \mathrm{F} 4 / 80^{+}$) analyzed by flow cytometry at day 12 to examine iNOS and Arg1 expression. Representative FACS profile of the expression (b). Representative graphical representation of one experiment $(\mathbf{c}) . N=5 . \mathbf{d}$ Tumor-infiltrating $\mathrm{CD} \mathrm{e}^{+} \mathrm{CD} 8^{+} \mathrm{T}$ cells purified by cell sorting at day 12, reactivated ex vivo, and Ifn $\gamma$ and Tnfo mRNA expression quantified by RT-qPCR, with mRNA expression level normalized by Rplp0 expression. $N=6$. e Cytotoxic T-lymphocyte degranulation assay. CD107a/LAMP1 expression on tumor-infiltrating CD8 ${ }^{+} \mathrm{T}$ cells was analyzed by flow cytometry after 12 days post-inoculation of B16-F10 cells. $N=8$ (ctrl), 9 (cKO) Gating: All cells>singlets $>$ live cells>CD8 ${ }^{+} \mathrm{T}_{\text {cell }>C D 107 a} \mathbf{f}$ NK cell degranulation assay by measuring CD107a/LAMP1 expression on tumor-infiltrating NK cells. $N=8$ (ctrl), 9 (cKO). Gating: All cells $>$ singlets $>$ live cells $>$ NK cells>CD107a. $\mathbf{g}$ CD4 ${ }^{+}$T cell degranulation assay by measuring CD107a/LAMP1 expression on CD4 ${ }^{+}$T cells. $N=8$. Gating: All cells $>$ singlets $>$ live cells $>$ CD4 ${ }^{+}$T cell>CD107a. h Flow cytometric analysis to show the changes in B16-F10 subcutaneous tumor-infiltrating Treg (CD4 ${ }^{+}$CD3e ${ }^{+}$FoxP3 ${ }^{+}$) populations upon Cyp11a1 deletion. $N=9$. i-I Comparing immunophenotype in Cyp11a1 inhibitor, AG, treated and untreated mice with B16-F10 cells injected subcutaneously. All are representative of two independent experiments. i Representative FACS profile to show iNOS and Arg1 expression in tumorinfiltrating $\mathrm{CD}_{4} 5^{+} \mathrm{Lin}^{-} \mathrm{CD} 11 \mathrm{~b}^{+} \mathrm{F} 4 / 80^{+}$macrophages (left panel). Graphical presentation the iNOS and Arg1 expression of a representative experiment (right panel). $N=5$. $\mathbf{j}-\mathbf{I}$ CD107a/LAMP1 expression on CD4 ${ }^{+} \mathrm{T}$ cells $(\mathbf{j}), \mathrm{CD} 8^{+} \mathrm{T}$ cells $(\mathbf{k})$ and NK cells $(\mathbf{I})$ was analyzed by flow cytometry after 12 days post-inoculation of B16-F10 cells. $N=4$ (ctrl), 3 (cKO). Gating: All cells>singlets>live cells>CD4+ or CD8 ${ }^{+}$T or NK cells>CD107a. In this figure, all error bars represent mean with s.d. $P$-value was calculated by unpaired two-tailed $t$-test. $N$ represents biologically independent animals.

receptors $^{53} \mathrm{PD} 1$ and TIGIT on tumor-infiltrating $\mathrm{T}$ cells was reduced in $\mathrm{CD}^{+}$TILs in the Cyp11a1 cKO mice compared with control littermates (Supplementary Fig. 5a). The data indicate greater $\mathrm{T}$ cell functionality and less exhaustion in the Cyp11a1 cKO mice.

To test cytotoxic capacity of $\mathrm{T}$ and NK cell populations, we examined the degranulation response of these cells by analyzing cell surface expression of CD107a/LAMP1 in tumor-infiltrating T and NK cells. We observed a significantly increased proportion of

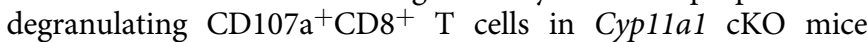
compared to control mice (Fig. 6e). The degranulation response in NK cells and CD4 ${ }^{+} \mathrm{T}$ cells was also enhanced (Fig. 6f, g).

Finally, we observed that the proportion of intratumoral Tregs was decreased (Fig. 6h). Altogether these data suggest that inhibition of T cell steroidogenesis by genetic deletion of Cyp11a1 changes immune cell composition in the tumor microenvironment in favor of anti-tumor immunity.

Similar to the genetic deletion of Cyp11a1, application of the Cyp1la1 inhibitor aminoglutethimide significantly increased frequencies of M1 macrophages and degranulating $\mathrm{T}$ and NK cells (Fig. 6i-1, Supplementary Fig. 5b), recapitulating anti-tumor phenotypes. Cyp11a1 inhibition also decreased the number of M2 macrophages in the tumor (Fig. 6i). Together, these data demonstrate the potential to stimulate anti-tumor immunity through pharmacological suppression of Cyp11a1-dependent steroidogenesis pathways.

\section{Discussion}

The endocrine importance of systemic steroid hormones is well documented in regulating cell metabolism and immune cell function but the intracrine, autocrine, and paracrine role of local cell-type-specific steroidogenesis is less clear, particularly in pathologies such as cancer ${ }^{1,2}$. This is in part due to the lack of tools to study steroidogenesis in a tissue-specific manner in vivo. To overcome this, we generated a Cyp11a1-mCherry reporter and a conditional Cyp11a1 knockout mouse strain to identify de novo steroidogenic cells and study their role in vivo. Using these discovery tools, complemented by pharmacological intervention, we uncovered an anti-tumor immune suppression mechanism that may be exploited clinically to boost the anti-tumor immunity (Fig. 7).

The TME harbors innate immune cells (such as macrophages, neutrophils, eosinophils, mast cells, myeloid derived suppressor cells, dendritic cells, and natural killer cells), adaptive immune cells (such as T and B cells), stromal cells (such as fibroblasts, endothelial cells, pericytes, mesenchymal cells), and cancer cells.
The presence of immune cells in the tumor microenvironment raises a long-standing question: How tumors evade the immune surveillance and anti-tumor immunity? The present understanding suggests that the balance between protective antitumoral immune cells (and other protective factors) and protumoral immunosuppressive cells (and other immunosuppressive factors) determines the fate. Anti-tumoral immune cell subsets includes Th1, Tc1 (CD8 ${ }^{+}$CTL), M1 macrophage, DC1 which are antagonized by Th2, M2, tolerogenic DC, immature DC, DC2, Treg, and myeloid-derived suppressor cells. Established tumors often foster immunosuppressive and tolerogenic immune cells. In this report, the discovery of immune cell-mediated steroid biosynthesis in the TME explains how an immunosuppressive TME can be achieved. Previously it has been shown that steroids induce anergy and cell death in $\mathrm{T}$ cells ${ }^{44-46,54}$, M2 phenotype in macrophages ${ }^{42,43}$, tolerance in $\mathrm{DCs}^{47-49}$, and increase the frequency of Treg cells ${ }^{50-52}$. Here we demonstrate that tumorinfiltrating $\mathrm{T}$ cells can produce steroids and that the inhibition of $\mathrm{T}$ cell-mediated steroid biosynthesis tips the balance toward protective immunity. We have shown that ablation of intratumoral steroids by deleting Cyp11a1 in T cells reduced the number of M2 macrophages and Tregs, and increased the number of M1 macrophages, functional T cells, and NK cells. The results suggest that the secreted steroid works in a paracrine manner that effect on other infiltrating immune cells as expected. All these cell types are known to be crucial in regulating anti-tumor immunity and immunosuppression. Therefore, in the absence of intratumoral steroids, anti-tumor immunity is more effective and restricts tumor progression. Importantly, this pathway can be targeted pharmacologically. This may be exploited therapeutically in three ways: (1) steroidogenic gene expression or the presence of steroids might be used to stratify the patients, (2) use of pharmacologic drug (i.e. anti-steroidogenic) can convert the TME into protective type- 1 inflammatory condition by reducing the steroid level, and (3) immunotherapy should work better in absence of immunosuppressive steroids.

Single-cell level transcriptomic analysis revealed important findings pertaining to tumor immune infiltrates and provide a rich resource for cancer immunologists. We identified three distinct classes of immune cells with steroidogenic potential, $\mathrm{T}$ cells (Th2 and Tc2, major population), mast cells (minor population), basophils (minor population), and a few monocyte/macrophages. GATA factors are revealed as key drivers of Cyp11al expression in immune cells. In Th2 cells Gata3 occupies the Cyp11a1 locus. Cyp11a1 ${ }^{+}$Th2 cells outnumbered other types of Cyp11a1expressing cells. Hence deletion of Cyp11a1 in $\mathrm{T}$ cells was 


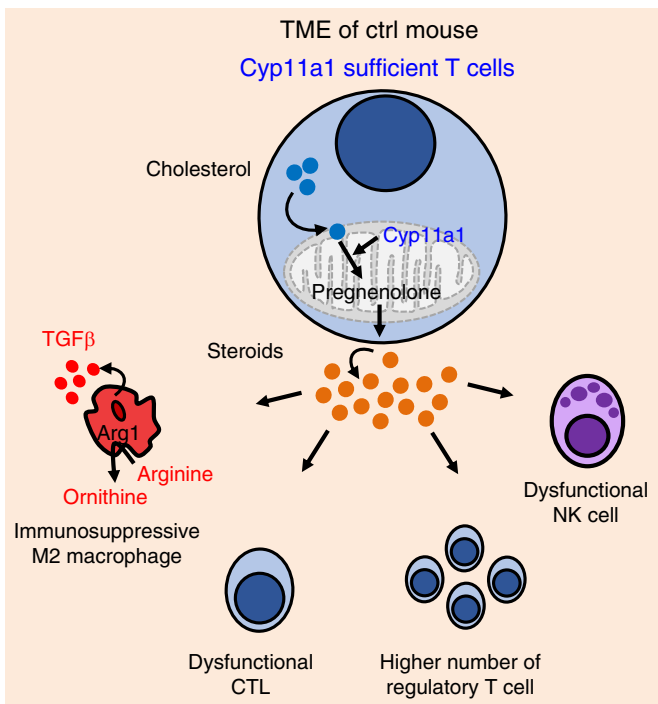

Tumor immune suppression

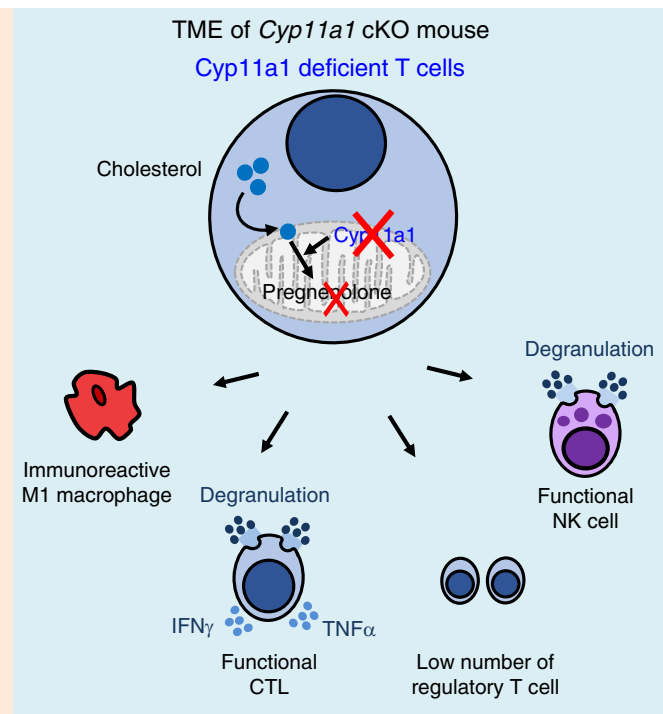

Tumor immune activation

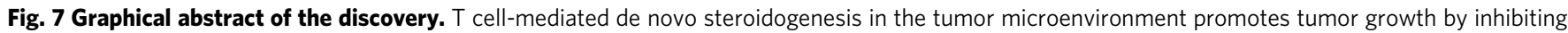
anti-tumor immunity, in part by inducing M2 phenotype in macrophages and suppressing T and NK cell function. Genetic deletion of Cyp11a1 or pharmacologic inhibition of Cyp11a1 activity stimulates anti-tumor immunity by increasing the number of $\mathrm{M} 1$ macrophages and functional $\mathrm{T}$ cells, and decreasing the number of regulatory T cells, M2 macrophages.

sufficient to remodel the TME for more effective anti-tumor immune responses. In the in vitro setting, we observed that Tregs and other $\mathrm{T}$ helper cell types (except Th1) can upregulate Cypllal expression, but in the in vivo setting we noticed that only Th2 cells were Cyp11a1+. We did not find Cyp11a1expressing Tregs or any other T helper cell types in the B16-F10 TME. The presence of Cyp1la1 ${ }^{+}$non-T cells indicates that in a different context, such as in different tumor types, the steroidogenic population may vary and other hematopoietic cells may also produce steroids. Future studies are essential to gain detailed insight of immune cell-mediated intratumoral steroidogenesis in different tumor type. It would be interesting to know why $\mathrm{CD} 4^{+}$ $\mathrm{T}$ cells upregulate Cyp11a1 expression specifically in the TME, and not in the secondary lymphoid organs. This could be a result of the fact that Th2 cells are numerous in tumors but not in the spleen and lymph nodes. Therefore, further studies are needed to delineate whether this is a result of an increased Th2 cell abundance in tumors, and/or an enhanced Cyp11a1 expression per individual Th2 cell.

The induction of type- 2 immune response in tumors such as breast, pancreatic, melanoma, glioma, cervical, lung, and bladder cancer has been previously reported, both in humans and mice, and its roles in the promotion of cancer by immunosuppression are known ${ }^{12-25}$. As expected, reanalysis of publicly available transcriptomic datasets showed evidence of many human tumor types expressing steroidogenic genes. In melanoma, we show evidence of steroidogenic gene expression being correlated with type- 2 immune signature genes such as IL4. The limitation of this analysis is that it does not confirm the cellular source of CYP11A1 expression because the transcriptomes were obtained from bulk RNAseq of whole tumors. In the future, single-cell RNA sequencing of tumor-infiltrating Th2 cells from patients may provide direct evidence and further insights. Analysis of publicly available single-cell RNA sequencing datasets of human tumorinfiltrating $\mathrm{T}$ cells revealed that all the available datasets are from tumors that predominantly raised Th1-mediated immune responses. Therefore, as expected, we observed no or only a few CYP11A1-expressing cells in these data sets (data not shown). This is consistent with our hypothesis in the sense that we expect
CYP11A1 expression predominantly in tumors exhibiting type-2 immune responses.

The study adds to the concern raised by recent studies about proper use of steroids, particularly glucocorticoids, in solid tumors as a part of the disease management, specifically when we seek to boost anti-tumor immune response ${ }^{55-62}$. Glucocorticoids have been used in clinical oncology for over a half a century, and at present these are routinely used to alleviate edema in patients with intracranial lesions and are first-line agents to suppress immune-related adverse events that arise with the immunotherapies. Therefore, further studies are required to evaluate the use of synthetic steroids if the tumors itself induces glucocorticoid synthesis. Reduction of intratumoral steroidogenesis may open a window for immunotherapies to work better at lower doses.

Similar experimental approaches can in future provide in depth mechanistic insights into other extraglandular (local) steroidogenic cell types such as adipose cells, neuron, osteoblasts, astrocytes, microglia, skin, trophoblast, and thymic epithelial cells. Our Cyp11a1-mCherry reporter mouse line can be used as a discovery tool to identify undiscovered steroidogenic cell types in tolerogenic physiological conditions such as pregnancy, mucosal tolerance, and inflammatory and immunopathological conditions. Their functional role can be dissected by using Cyp11a1 cKO mice using tissue-specific Cre-drivers. Nevertheless, cancer is a proven context where tumors hijack this pathway for immune evasion. Further studies in diverse physiological scenarios would be of great interest to more broadly understand the role of immune cell de novo steroidogenesis in regulating inflammation and immunity.

\section{Methods}

Mice. The use of all mice in this research article were in accordance with the UK Animals in Science Regulation Unit's Code of Practice for the Housing and Care of Animals Bred, Supplied or Used for Scientific Purposes, the Animals (Scientific Procedures) Act 1986 Amendment Regulations 2012. All experimental procedures were performed under UK Home Office Project licenses (PPL 80/2574 or PPL P8837835 or PPL P6B8058B0), which were reviewed and approved by the Medical Research Council Laboratory of Molecular Biology (PPL 80/2574 or PPL

P8837835) and Wellcome Sanger Institute (PPLP6B8058B0) Animal Welfare and 
Ethical Review Bodies (AWERB). Sample sizes were determined based on previous experience and a priori power analysis ( $\mathrm{G}^{*}$ Power). Housing conditions at the Sanger Institute: all the mice used in this study, were maintained in specific pathogen-free unit on a 12-h light and 12-h dark cycle (lights off at 7:30 pm and no twilight period). The ambient temperature was $21^{\circ} \mathrm{C}$ with a maximum variation of $\pm 2{ }^{\circ} \mathrm{C}$. The humidity was $55 \pm 10 \%$. Mice were housed for phenotyping with a stocking density of 3-5 mice/cage (dimensions of caging: $(\mathrm{L} \times \mathrm{W} \times \mathrm{H}) 365 \times 207 \times$ $140 \mathrm{~mm}$, floor area $530 \mathrm{~cm}^{2}$ ) in individually ventilated caging (Tecniplast Seal Safe1284L) receiving 60 air changes/h. In addition to Aspen bedding substrate, we provide standard environmental enrichment of two nestlets, a cardboard Fun Tunnel and three wooden chew blocks. Given water and diet were available all the times. Mice were fed on Mouse Breeders Diet (Lab Diets, 5021-3). Animals recruited to studies at ARES Medical Research Council animal facility remained socially housed in individually ventilated cages, at ambient temperature and with cage enrichment. Animals of each genotype were randomly assigned to experimental groups, and where possible, technicians performing the experiment were blinded to experimental groups and treatments.

Generation of a Cyp11a1-mCherry reporter mouse line. Using CRISPR_Cas9 technology we introduced double-strand DNA breaks $5^{\prime}$ and $3^{\prime}$ adjacent to the Cyp11al termination codon in exon 9 to facilitate the introduction of our targeting construct. The $5^{\prime}$ and $3^{\prime}$ arms of homology were designed to remove the Cyp11al termination codon and $100 \mathrm{bp}$ of the $3^{\prime}$ UTR immediately downstream and replace it with a minimal T2a self-cleavage peptide followed by the fluorescent marker mCherry. Using the web-based tool designed by Hodgkins et al. ${ }^{63}$, two sgRNAs were identified $5^{\prime}$ and $3^{\prime}$ adjacent to the Cyp1lal termination codon. The guide sequences were ordered from Sigma Genosys as sense and antisense oligonucleotides, and annealed before individually cloning into a human U6 (hU6) expression plasmid (Addgene \# 41824). Next, we generated targeted ES cell (ESCs) through nucleofection of $3 \times 10^{6} \mathrm{JM} 8$ ESCs with $2 \mu \mathrm{g}$ Cyp 11 al circular targeting construct, $1.5 \mu \mathrm{g}$ of each hU6_sgRNAs, and $3 \mu \mathrm{g}$ a plasmid expressing human codonoptimized CAS9 driven by with the CMV promoter (Addgene \# 41815). $48 \mathrm{~h}$ post transfection the media was changed for G418 selection media (165 ug/ul). Confirmation of the correct targeting events were confirmed by quantitative PCR (qPCR) for loss of heterozygosity (LOH) and the presence of the selectable marker, and long range (LR) PCR and Sanger sequencing. Following blastocyst injection and chimaera breeding three F1 Cyp11a1+/mCherry-Neo mice were bred to pCAGGSs-Flp ${ }^{64}$ mice to remove the neomycin selectable marker to generate

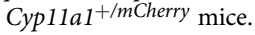

Genotyping primer sequence (Supplementary Fig. 1a)

GF ATGGTGACACAGACCTCCTTG

GR CCCAGGAACAGAAAGGCTTG

R2R AGGCGCATAACGATACCACG

R1R GGATTCTCCTCCACGTCACCG

Generation of a Cyp11a1 cKO mice. Cyp11a1 $1^{f l f l}$ mice were generated by crossing Cyp11a1 $1^{\text {tmla }}(\mathrm{KOMP})$ Wtsi mice with a previously reported Flp-deleter (FlpO) line ${ }^{64}$. Cyp11al flfl mice were crossed with Cd4-cre mice to generate the Cyp11al cKO mice.

Syngeneic mouse tumor models. B16-F10 melanoma model: the C57BL/6 derived B16-F10 melanoma cell line was purchased from American Type Culture Collection (ATCC) and cultured in Dulbecco's Modified Eagle medium (DMEM, Life Technologies), supplemented with $100 \mathrm{U} / \mathrm{mL}$ penicillin/streptomycin and $10 \%$ fetal bovine serum (FBS; Life Technologies, Invitrogen). For the primary tumor growth assay, $2.5 \times 10^{5} \mathrm{~B} 16$-F10 cells were injected subcutaneously into the shoulders of either wild type (WT) C57BL/6 mice, Cd4-Cre, Cyp11a $f^{f l / f l}$ or Cd4$C r e ; C y p 11 a 1^{f l f l}$ mice. Once tumors were palpable, non-invasive tumor measurements were recorded daily and volumes calculated using the following formula $(\pi / 6)$ (shortest length $\times$ longest length). After 11 or 12 days animals were killed and tissues collected for analysis. For the experimental metastasis assay, $5 \times 10^{5} \mathrm{~B} 16-$ F10 cells in a volume of $0.1 \mathrm{ml}$ PBS were injected intravenously into the tail vein. After 10 days ( \pm 1 day) the mice were killed via cervical dislocation, and their lungs removed and rinsed in phosphate-buffered saline. The number of B16-F10 colonies on all five lobes of the lung were counted macroscopically. Exogenous pregnenolone treatment: On days 5, 7, and 9, tumor-bearing mice received $10 \mu \mathrm{l}$ of vehicle or pregnenolone $(20 \mathrm{mg} / \mathrm{ml})$ in DMSO via topical application at tumor site. Tumor volumes were recorded until the experimental endpoint on day 12. Orthotopic EO771 breast cancer model: the EO771 breast cancer cell line was purchased from CH3 BioSystems and cultured in RPMI (Sigma) supplemented with 10\% FBS, $1 \%$ PS, and $10 \mathrm{mM}$ HEPES. In all, $2.5 \times 10^{5}$ cells were injected into the 4 th inguinal mammary fat pad of Cyp11a1-mCherry reporter mice. Tumors and inguinal lymph node were collected after 15 days of tumor development.

Lung colonization of EO771 cells: $4 \times 10^{5}$ cells were injected intravenously in to the tail vein. After 10 days ( \pm 1 day) the mice were killed via cervical dislocation, and their lungs were removed, rinsed in PBS and fixed in $10 \%$ neutral buffered formalin. The fixed lungs were paraffin embedded and sections stained with Hematoxylin and Eosin using routine histology protocols. The microscopic images were captured in Olympus with $\times 10$ magnification and the number of metastatic foci were counted. Treatment with aminoglutethimide (AG): for both the B16-F10 and E0771 models $200 \mu \mathrm{l}$ of a $2-\mathrm{mg} / \mathrm{ml} \mathrm{AG}$ or vehicle were administered via the intraperitoneal route. The first dose was administered concurrently with cell implantation and repeated every 2 days thereafter.

Tumor tissue processing. Tumors were mechanically dissociated and digested in $1 \mathrm{mg} / \mathrm{ml}$ collagenase D (Roche), $1 \mathrm{mg} / \mathrm{ml}$ collagenase A (Roche), and $0.4 \mathrm{mg} / \mathrm{ml}$ DNase I (Sigma) in IMDM media containing $10 \% \mathrm{FBS}$, at $37^{\circ} \mathrm{C}$ for 40 mins. EDTA was added to all samples to neutralize collagenase activity (final concentration 5 $\mathrm{mM}$ ) and digested tissues were passed through $70 \mu \mathrm{m}$ filters (Falcon)

Cell sorting. Once processed, single-cell suspension tumor samples were incubated with a fixable fluorescent viability stain (Life Technologies) for 20 mins (diluted 1:1000 in PBS) prior to incubation with conjugated primary antibodies for 30 mins at $4{ }^{\circ} \mathrm{C}$. Antibodies were diluted in PBS with $0.5 \%$ bovine serum albumin (BSA). Stained samples were sorted, using the MoFlo XDP or BD Influx cytometer system.

T helper cell culture. Splenic naïve T helper cells from Cyp11a1-mCherry reporter mice were purified with the $\mathrm{CD} 4{ }^{+} \mathrm{CD} 62 \mathrm{~L}^{+} \mathrm{T}$ Cell Isolation Kit II (Miltenyi Biotec) and polarized in vitro toward differentiated Th1, Th2, Th9, Th17, iTreg, and Tfh subtype following Pramanik et al. ${ }^{65}$. Naïve cells were seeded into anti-CD3e $(2 \mu \mathrm{g} /$ $\mathrm{ml}$, clone $145-2 \mathrm{C} 11$, eBioscience $)$ and anti-CD28 $(5 \mu \mathrm{g} / \mathrm{ml}$, clone 37.51 ,

eBioscience) coated plates. The medium contained the following cytokines and/or antibodies as described below. To culture Th1 subtype: recombinant murine IL2 (10 ng/ml, R\&D Systems), recombinant murine IL12 (10 ng/ml, R\&D Systems), and neutralizing anti-IL4 $(10 \mu \mathrm{g} / \mathrm{ml}$, clone 11B11, eBioscience). Th2 subtype: Recombinant murine IL2 ( $10 \mathrm{ng} / \mathrm{ml}$, R\&D Systems), recombinant murine IL-4 (10 ng/ml, R\&D Systems), and neutralizing anti-IFNg $(10 \mu \mathrm{g} / \mathrm{ml}$, clone XMG1.2, eBioscience). Th9 subtype: $20 \mathrm{ng} / \mathrm{ml}$ recombinant mouse IL $4,2 \mathrm{ng} / \mathrm{ml}$ recombinant

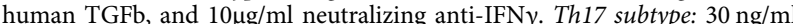
recombinant mouse IL6, $5 \mathrm{ng} / \mathrm{ml}$ recombinant human TGFb, and $50 \mathrm{ng} / \mathrm{ml}$ recombinant mouse IL23. Tfh subtype: $50 \mathrm{ng} / \mathrm{ml}$ recombinant mouse IL21, $10 \mu \mathrm{g} /$ $\mathrm{ml}$ neutralizing anti-IL4 and anti-IFN $\gamma$. iTreg subtype: $5 \mathrm{ng} / \mathrm{ml}$ recombinant mouse IL2, $5 \mathrm{ng} / \mathrm{ml}$ recombinant human TGFb. The cells were removed from the activation plate on day 4 (after $72 \mathrm{~h}$ ). Th2 cells were cultured for another 2 days in the absence of CD3e and CD28 stimulation. Then, cells were restimulated by seeding on coated plate for $6 \mathrm{~h}$. For flow cytometric detection cells were treated with monensin $(2 \mu \mathrm{M}$, eBioscience) for the last $3 \mathrm{~h}$.

In vitro Tc1 and Tc2 differentiation. Splenic naïve $\mathrm{CD} 8^{+} \mathrm{T}$ cells were purified by using Naive CD8 $\mathrm{a}^{+} \mathrm{T}$ Cell Isolation Kit, mouse (Miltenyi Biotec) following the manufacturers protocol, and polarized in vitro toward differentiated Tc1 and $\mathrm{Tc} 2$. In brief, naive cells were seeded into anti-CD3e $(2 \mu \mathrm{g} / \mathrm{ml}$, clone $145-2 \mathrm{C} 11$, eBioscience) and anti-CD28 (5 $\mu \mathrm{g} / \mathrm{ml}$, clone 37.51, eBioscience) coated plates. The medium contained the following cytokines and/or antibodies:

Tc1 subtype: recombinant murine IL2 $(10 \mathrm{ng} / \mathrm{ml}, \mathrm{R} \& D$ Systems), recombinant murine IL12 $(10 \mathrm{ng} / \mathrm{ml}, \mathrm{R} \& D$ Systems $)$ and neutralizing anti-IL4 $(10 \mu \mathrm{g} / \mathrm{ml}$, clone 11B11, eBioscience). Tc2 subtype: Recombinant murine IL2 (10 ng/ml, R\&D Systems), recombinant murine IL-4 (10 ng/ml, R\&D Systems) and neutralizing anti-IFNg $(10 \mu \mathrm{g} / \mathrm{ml}$, clone XMG1.2, eBioscience).

Single-cell RNA sequencing. Single cells were isolated from processed B16-F10 subcutaneous tumors using a fluorescence-activated cell sorter. Once processed, tumor tissues were incubated with a fixable fluorescent viability stain (Live-Dead Violet, Life Technologies) for 15 mins (diluted 1:1000 in PBS) prior to incubation with conjugated primary antibodies for $30 \mathrm{mins}$ at $4^{\circ} \mathrm{C}$ in the dark. Antibodies were diluted in flow cytometry staining buffer (eBioscience/Thermo Fisher Scientific). After staining, samples were sorted using the $\mathrm{BD}$ influx flow cytometer. Single-cells were harvested in $2 \mu \mathrm{l}$ of Lysis Buffer (1:20 solution with RNase Inhibitor (Clontech, cat. no. $2313 \mathrm{~A}$ ) in $0.2 \% \mathrm{v} / \mathrm{v}$ Triton X-100 (Sigma-Aldrich, cat. no. T9284)) in 96-well plates. After sorting the plates were spun down quickly and kept frozen at $-80^{\circ} \mathrm{C}$. Then we performed reverse transcription (RT) and cDNA pre-amplification following SmartSeq2 protocol to obtain mRNA libraries from single-cells. Oligo-dT primer, dNTPs (ThermoFisher, cat. no. 10319879) were then added to the samples. RT-PCR were performed using 50U of SMARTScribe Reverse Transcriptase (Clontech, cat. no. 639538). cDNA libraries were prepared using the Nextera XT DNA Sample Preparation Kit (Illumina, cat. no. FC-1311096), following the protocol supplied by Fluidigm (PN 100-5950 B1). Single-cell libraries were pooled, purified using AMPure XP beads (Beckman Coulter) and sequenced on an Illumina HiSeq 2500 aiming for an average depth of 1 million reads/cell (paired-end 100-bp reads).

Single-cell RNA sequencing analysis. The sequencing data was mapped using STAR 2.5.1b and quantified using htseq 0.9.0. The data was subsequently processed in Scanpy 1.3.6 with the standard Seurat-inspired workflow. Cells with above $20 \%$ mitochondrial reads were removed, and the data was transformed to $\log (\mathrm{CPM} / 100$ +1 ) form. Highly variable genes were identified via normalized dispersion, with the top $10 \%$ of genes reported as variable. The data was filtered to highly variable 
genes, scaled and used for PCA. The top 20 principal components were used to infer a neighbor graph, which served as the basis of UMAP dimensionality reduction and Leiden clustering with a 0.4 resolution value. Per-cluster markers were identified using default Scanpy settings, using $\log (\mathrm{CPM} / 100+1)$ data on input. Genes correlated with Cyp11a1 were identified with the Pearson Correlation Coefficient. Potential Cyp11a1 regulators were detected via pySCENIC, replicating the demonstration notebook.

ATAC-seq. Naïve T cells isolation and in vitro $\mathrm{T}$ helper cell differentiation were carried out as described above. After $72 \mathrm{~h}$ of continuous activation/differentiation, Th1, Th2, and Th17 cells were harvested. The ATAC-seq experiments were performed following the published protocol with some modification ${ }^{66}$. Briefly, 50,000 cells were washed with ice-cold 1X Dulbecco's PBS (twice), and resuspended in a sucrose swelling buffer $(0.32 \mathrm{M}$ sucrose, $10 \mathrm{mM}$ Tris.Cl, $\mathrm{pH} 7.5,3 \mathrm{mM} \mathrm{CaCl}, 2$ $\mathrm{mM} \mathrm{MgCl}, 10 \%$ glycerol). The cell suspension was incubated for $10 \mathrm{~m}$ on ice. In next, a final concentration of $0.5 \% \mathrm{NP}-40$ was added, and the cells suspension was vortexed for $10 \mathrm{~s}$ and incubated for $10 \mathrm{~m}$ on ice. Nuclei were pelleted at $500 \times g$ at $4{ }^{\circ} \mathrm{C}$ for $10 \mathrm{~m}$. Then, the nuclei pellets were washed once with $1 \mathrm{X}$ TD buffer (from Nextera DNA Library Preparation Kit, Illumina, \#FC-121-1030), and resuspended in $50 \mathrm{ul}$ tagmentation mixture that contains $25 \mathrm{ul} 2 \mathrm{X}$ TD buffer (Nextera DNA Library Preparation Kit, Illumina \#FC-121-1030), 22.5 ul H2O, 2.5 ul TDE1 (Nextera DNA Library Preparation Kit, Illumina \#FC-121-1030). The tagmentation reaction was performed on an Eppendorf ThermoMixer C at $37^{\circ} \mathrm{C}, 800 \mathrm{rpm}$, for $30 \mathrm{~m}$. The reaction was stopped by adding $250 \mu \mathrm{l}(5 \mathrm{vol})$ Buffer PB (QIAGEN MinElute PCR Purification Kit). The tagmented DNA was purified by QIAGEN PCR Purification Kit according to manufacturer's instructions and eluted in $12.5 \mu \mathrm{l}$ Buffer EB from the kit, which yielded $\sim 10 \mu$ purified DNA. The library amplification was performed in a $25-\mu \mathrm{l}$ reaction that includes $10 \mu \mathrm{l}$ purified DNA (from above), 2.5 $\mu$ l PCR Primer Cocktail (Nextera DNA Library Preparation Kit, Illumina \#FC-121-1030), $2.5 \mu \mathrm{l} \mathrm{N5xx} \mathrm{(Nextera} \mathrm{index} \mathrm{kit,} \mathrm{Illumina} \mathrm{\# FC-121-1012),}$ $2.5 \mu \mathrm{l}$ N7xx (Nextera index kit, Illumina \#FC-121-1012), and 7.5 $\mu \mathrm{l}$ NPM PCR master mix (Nextera DNA Library Preparation Kit, Illumina \#FC-121-1030). PCR reaction condition was: $72^{\circ} \mathrm{C} 5 \mathrm{~m}, 98^{\circ} \mathrm{C} 2 \mathrm{~m},\left[98^{\circ} \mathrm{C} 10 \mathrm{~s}, 63^{\circ} \mathrm{C} 30 \mathrm{~s}, 72^{\circ} \mathrm{C} 60 \mathrm{~s}\right] \times$ $12,10^{\circ} \mathrm{C}$ hold. Amplified libraries were purified by double Agencourt AMPureXP beads purifications (Beckman Coulter, \#A63882). 0.4X beads:DNA ratio for the first time, flow through was kept (removing large fragments); 1.4X beads: DNA ratio for the second time, beads were kept. Libraries were eluted from the beads in $20 \mu \mathrm{l}$ Buffer EB (from QIAGEN PCR Purification Kit). In all, $1 \mu \mathrm{l}$ library was run on an Agilent Bioanalyzer to check size distribution and quality of the libraries. Sequencing was done by an Illumina Hiseq 2500 (75 bp PE).

ATAC-seq data processing. ATAC-seq reads were aligned using Bowtie $2^{67}$ with the parameter -X 2000 and the mouse genome $\mathrm{mm} 10$. This was followed by peak calling on each replicate individually using MACS ${ }^{68}$ with the function callpeak and the parameters -B -SPMR -shift -100 -extsize 200. The peaks obtained were kept if they overlapped a peak from the other replicate of the same T helper cell types. The resulting bedGraph files generated by MACS2 were converted to bigWig format for the visualization on UCSC genome browser ${ }^{69}$.

ChIP-seq data processing. GATA3 ChIP-seq data sets from different Tn subtypes were downloaded from the NCBI GEO database (GSE20898) ${ }^{70}$. The SRA files were converted to fastq files using the SRA toolkits (http://ncbi.github.io/sra-tools/). Reads were aligned to the mm10 genome using Bowtie $2^{67}$ with default parameters. Peak calling was performed by using MACS2 ${ }^{68}$ with the function callpeak and the parameters -B -SPMR -nomodel -extsize 200. The resulting bedGraph files generated by MACS2 were converted to bigWig format for the visualization on UCSC genome browser ${ }^{69}$.

B16-F10 and T cell co-culture assay. Negatively purified splenic naïve CD4 ${ }^{+}$ $\mathrm{T}$ cells from Cyp11a1-mCherry mice were cultured in presence of B16-F10 cell with or without TCR activation and analyzed by flow cytometry to detect Cyp11a1mCherry expression. Naïve $\mathrm{T}$ cell purification and activation condition is described in the T helper cell culture section above. Briefly, naïve splenic cells were activated in anti-CD3e and anti-CD28 coated plate in presence of IL2 but absence anti-IL4 neutralizing antibody.

Quantitative PCR. Tumor-infiltrating macrophages $\left(\mathrm{Lin}^{-} \mathrm{CD} 11 \mathrm{~b}^{+}\right)$and $\mathrm{CD} 8{ }^{+}$ $\mathrm{T}$ cells were purified by cell sorting. We used the Cells-to- $\mathrm{C}_{\mathrm{T}}$ kit (Invitrogen/ Thermofisher Scientific) and followed SYBR Green format according to manufacturer's instructions. In all, $2 \mu \mathrm{l}$ of cDNA was used in $12 \mu \mathrm{l}$ qPCR reactions with appropriate primers and SYBR Green PCR Master Mix (Applied Biosystems). Data were analyzed by ddCT method. Experiments were performed three times and data represent mean values \pm standard deviation. The primer list is provided below:

Arg1: F- ATGGAAGAGACCTTCAGCTAC R- GCTGTCTTCCCAAGAGTTGGG

Tgfß1: F- TGACGTCACTGGAGTTGTACGG R- GGTTCATGTCATGGATGGTGC

Ifn $\gamma$ : F- ACAATGAACGCTACACACTGC
R- CTTCCACATCTATGCCACTTGAG;

Tnf $\alpha$ : F- CATCTTCTCAAAATTCGAGTGACAA

R- TGGGAGTAGACAAGGTACAACCC

Gapdh: F- ACCACAGTCCATGCCATCAC

R- GCCTGCTTCACCACCTTC

Rplp0: F: CACTGGTCTAGGACCCGAGAA

R: GGTGCCTCTGGAGATTTTCG

Flow cytometry. We followed eBioscience surface staining, intracellular cytotoplasmic protein staining (for cytokines) protocols. Briefly, single-cell suspensions were stained with Live/Dead Fixable Dead cell stain kit (Molecular Probes/ Thermo Fisher) and blocked by purified rat anti-mouse CD16/CD32 purchased from BD Bioscience and eBioscience. Surface staining was performed in flow cytometry staining buffer (eBioscience) or in PBS containing 3\% FCS at $4{ }^{\circ} \mathrm{C}$. For intracellular cytokine staining cells were fixed by eBioscience IC Fixation buffer and permeabilized by eBioscience permeabilization buffer. Cells were stained in $1 \mathrm{x}$ permeabilization buffer with fluorescent dye-conjugated antibodies. After staining cells were washed with flow cytometry staining buffer (eBioscience) or 3\% PBS-FCS, and were analyzed by flow cytometer Fortessa (BD Biosciences) using FACSDiva. The data were analyzed by FlowJo v10.2 software. Antibodies used in flow cytometry were: CD4 (RM4-5 or GK1.5), eBioscience, BD Bioscience and Biolegend. 1:400; CD8a (53-6.7), eBioscience 1:400; CD3e (145 2c11), eBioscience 1:400; TCRb (H57-597, BB790) BD Bioscience 1:400; CD45 (30F11), BD Bioscience 1:1000; CD44 (IM7), eBioscience 1:400; CD25 (PC61), BD Bioscience 1:400; B220 (Ra36b2), eBioscience 1:400; Ly6G (1A8), eBioscience 1:400; Ly6G/Ly6C/Gr-1 (RB68C5), eBioscience 1:400; Ly6C (HK1.4), eBioscience 1:400; SiglecF (E50-2440) BD Bioscience 1:1000; CD11b (M1/70), eBioscience 1:400; CD11c (N418), eBioscience 1:400; CD19 (1D3), eBioscience 1:400; NK1.1 (Pk136), Biolegend 1:400; Ter119 (TER119), eBioscience 1:400; PD-1 (J43), eBioscience 1:400; TIGIT (1G9), BD Bioscience 1:400; CD107a/LAMP1 (1D4B), eBioscience $0.5 \mu \mathrm{g} /$ test(100ul); IFNg (XMG1.2) PerCP-Cy5.5 eBioscience 1:1500 Cat no. 45-7311-82; IL4 (11B11) APC eBioscience 1:200 Cat no 17-7041-82; IL13 (eBio13a) AF488 eBioscience, 1:400, Cat. no. 53-7133-82; IL17 (eBio64DEC17), APC, 1:200, Cat. no. 17-7179-42; CD117(2B8) BV711, BD Bioscience, cat no. 563160.

Western blot antibodies. Anti-CYP11A1 (Santa Cruz Biotechnology, C-16) and anti-TBP (Abcam) were used.

Quantitative ELISA. CD45 leukocytes were purified from B16-F10 tumor masses and lungs, of mice that had been tail vein administered B16-F0 cells, and seeded at equal density in IMDM medium supplemented with $10 \%$ charcoal stripped FBS (Life Technologies, Invitrogen) for $24 \mathrm{~h}$. Pregnenolone concentrations of the culture supernatants were quantified using pregnenolone ELISA kit (Abnova) and corticosteroids ELISA (Thermofisher) kit following the manufacturers' instruction Absorbance was measured at $450 \mathrm{~nm}$, and data were analyzed in GraphPad Prism 6.

T cell proliferation assay. Negatively purified splenic naïve $\mathrm{CD} 4^{+} \mathrm{T}$ cells were purified from Cyp11a1 cKO, Cd4-Cre and Cyp11a $1^{f / / l}$ mice, stained with CellTrace Violet following the CellTrace Violet Cell Proliferation Kit (Invitrogen) protocol, activated in vitro, and the cell proliferation profile was captured by flow cytometry based dye decay assay on BD Fortessa ${ }^{65}$. Data were analyzed in FlowJo v9.

Reporting summary. Further information on research design is available in the Nature Research Reporting Summary linked to this article.

\section{Data availability}

scRNAseq data is deposited to ArrayExpress with accession number E-MTAB-8509 [https://www.ebi.ac.uk/arrayexpress/experiments/E-MTAB-8509/]. The ATACseq data (exclusively generated for this study) is in ENA and deposited within a large study name with many other data sets. The accession number in ENA is PRJEB14081 [https://www. ebi.ac.uk/ena/data/search?query=PRJEB14081]. The sample info and run numbers referring to the ATAC-seq data we used in the manuscript can be found in the spreadsheet provided as Supplementary Data 1. GATA3 ChIP-seq data sets from different Tn subtypes were downloaded from the NCBI GEO database (GSE20898) [https://www.ncbi.nlm.nih.gov/geo/query/acc.cgi?acc=GSE20898]. The raw data source of Fig. 3j was GSE19234 ${ }^{71}$ [https://www.ncbi.nlm.nih.gov/geo/query/acc.cgi? acc $=$ GSE19234] and Fig. $2 \mathrm{l}$ was EGAD00001000325 ${ }^{72}$ [https://www.ebi.ac.uk/ega/ datasets/EGAD00001000325]. Access to EGAD00001000325 dataset is available upon request to the Data Access Committee at datasharing@sanger.ac.uk. In Supplementary Fig. $2 \mathrm{~g}$ we searched "Expression Atlas (EMBL-EBI)" with search query gene name "Cyp1la1", species "Homo sapiens", "cancer" as disease condition, baseline expression, arranged by expression rank, downloaded data, rebuilt the figure, excluded ovarian cancer to avoid confusion. Link for the search is provided below: https://www.ebi.ac.uk/ gxa/search?geneQuery=\%5B\%7B\%22value $\% 22 \% 3 \mathrm{~A} \% 22$ Cyp 11 a $1 \% 22 \% 7 \mathrm{D} \%$ $5 \mathrm{D} \&$ species $=$ homo $\% 20$ sapiens\&conditionQuery $=\% 5 \mathrm{~B} \% 7 \mathrm{~B} \% 22$ value $\% 22 \% 3 \mathrm{~A} \%$ 22cancer\%22\%7D\%5D\&bs=\%7B\%22homo\%20sapiens\%22\%3A\%5B\%22DISEASE $\% 22 \%$ 5D\%7D\&ds=\%7B\%22kingdom\%22\%3A\%5B\%22animals\%22\%5D\%7D\#baseline. 
Supporting data for the Supplementary Fig. $4 \mathrm{j}-\mathrm{i}$ can be found in the Supplementary Data 2. All remaining relevant data are available in the article, supplementary information, or from the corresponding author upon reasonable request.

Received: 5 April 2020; Accepted: 22 June 2020;

Published online: 17 July 2020

\section{References}

1. Miller, W. L. \& Auchus, R. J. The molecular biology, biochemistry, and physiology of human steroidogenesis and its disorders. Endocr. Rev. 32, 81-151 (2011).

2. Miller, W. L. Steroidogenesis: unanswered questions. Trends Endocrinol. Metab. 28, 771-793 (2017).

3. Belelli, D. \& Lambert, J. J. Neurosteroids: endogenous regulators of the GABA (A) receptor. Nat. Rev. Neurosci. 6, 565-575 (2005).

4. Slominski, A. et al. Steroidogenesis in the skin: implications for local immune functions. J. Steroid Biochem. Mol. Biol. 137, 107-123 (2013).

5. Vacchio, M. S., Papadopoulos, V. \& Ashwell, J. D. Steroid production in the thymus: implications for thymocyte selection. J. Exp. Med. 179, 1835-1846 (1994).

6. Li, J., Papadopoulos, V. \& Vihma, V. Steroid biosynthesis in adipose tissue. Steroids 103, 89-104 (2015).

7. Cima, I. et al. Intestinal epithelial cells synthesize glucocorticoids and regulate T cell activation. J. Exp. Med. 200, 1635-1646 (2004).

8. Hostettler, N. et al. Local glucocorticoid production in the mouse lung is induced by immune cell stimulation. Allergy 67, 227-234 (2012).

9. Hanahan, D. \& Weinberg, R. A. Hallmarks of cancer: the next generation. Cell 144, 646-674 (2011)

10. Vinay, D. S. et al. Immune evasion in cancer: mechanistic basis and therapeutic strategies. Semin Cancer Biol. 35, S185-S198 (2015).

11. Beatty, G. L. \& Gladney, W. L. Immune escape mechanisms as a guide for cancer immunotherapy. Clin. Cancer Res. 21, 687-692 (2015).

12. DeNardo, D. G. et al. CD4(+) T cells regulate pulmonary metastasis of mammary carcinomas by enhancing protumor properties of macrophages. Cancer Cell 16, 91-102 (2009).

13. Kobayashi, M., Kobayashi, H., Pollard, R. B. \& Suzuki, F. A pathogenic role of Th2 cells and their cytokine products on the pulmonary metastasis of murine B16 melanoma. J. Immunol. 160, 5869-5873 (1998).

14. De Monte, L. et al. Intratumor T helper type 2 cell infiltrate correlates with cancer-associated fibroblast thymic stromal lymphopoietin production and reduced survival in pancreatic cancer. J. Exp. Med. 208, 469-478 (2011).

15. Hanahan, D. \& Coussens, L. M. Accessories to the crime: functions of cells recruited to the tumor microenvironment. Cancer Cell 21, 309-322 (2012).

16. Disis, M. L. Immune regulation of cancer. J. Clin. Oncol. 28, 4531-4538 (2010).

17. Sinha, P., Clements, V. K., Bunt, S. K., Albelda, S. M. \& Ostrand-Rosenberg, S. Cross-talk between myeloid-derived suppressor cells and macrophages subverts tumor immunity toward a type 2 response. J. Immunol. 179, 977-983 (2007).

18. Protti, M. P. \& De Monte, L. Cross-talk within the tumor microenvironment mediates Th2-type inflammation in pancreatic cancer. Oncoimmunology 1, 89-91 (2012).

19. Shiao, S. L., Ganesan, A. P., Rugo, H. S. \& Coussens, L. M. Immune microenvironments in solid tumors: new targets for therapy. Genes Dev. 25, 2559-2572 (2011).

20. Maeda, H. \& Shiraishi, A. TGF-beta contributes to the shift toward Th2-type responses through direct and IL-10-mediated pathways in tumor-bearing mice. J. Immunol. 156, 73-78 (1996).

21. Sheu, B. C. et al. Predominant Th2/Tc2 polarity of tumor-infiltrating lymphocytes in human cervical cancer. J. Immunol. 167, 2972-2978 (2001).

22. Medler, T. R., Cotechini, T. \& Coussens, L. M. Immune response to cancer therapy: mounting an effective antitumor response and mechanisms of resistance. Trends Cancer 1, 66-75 (2015).

23. Aspord, C. et al. Breast cancer instructs dendritic cells to prime interleukin 13secreting CD4+ T cells that facilitate tumor development. J. Exp. Med. 204, 1037-1047 (2007).

24. Pedroza-Gonzalez, A. et al. Thymic stromal lymphopoietin fosters human breast tumor growth by promoting type 2 inflammation. J. Exp. Med. 208, 479-490 (2011).

25. Coussens, L. M., Zitvogel, L. \& Palucka, A. K. Neutralizing tumor-promoting chronic inflammation: a magic bullet? Science 339, 286-291 (2013).

26. Cain, D. W. \& Cidlowski, J. A. Immune regulation by glucocorticoids. Nat. Rev. Immunol. 17, 233-247 (2017).
27. Klein, S. L. \& Flanagan, K. L. Sex differences in immune responses. Nat. Rev. Immunol. 16, 626-638 (2016).

28. Mahata, B. et al. Single-cell RNA sequencing reveals $\mathrm{T}$ helper cells synthesizing steroids de novo to contribute to immune homeostasis. Cell Rep. 7, 1130-1142 (2014).

29. Skarnes, W. C. et al. A conditional knockout resource for the genome-wide study of mouse gene function. Nature 474, 337-342 (2011).

30. Mellman, I., Coukos, G. \& Dranoff, G. Cancer immunotherapy comes of age Nature 480, 480-489 (2011)

31. Landskron, G., De la Fuente, M., Thuwajit, P., Thuwajit, C. \& Hermoso, M. A. Chronic inflammation and cytokines in the tumor microenvironment. J. Immunol. Res. 2014, 149185 (2014).

32. Papatheodorou, I. et al. Expression Atlas: gene and protein expression across multiple studies and organisms. Nucleic acids Res. 46, D246-D251 (2018).

33. Curran, M. A., Montalvo, W., Yagita, H. \& Allison, J. P. PD-1 and CTLA-4 combination blockade expands infiltrating $\mathrm{T}$ cells and reduces regulatory $\mathrm{T}$ and myeloid cells within B16 melanoma tumors. Proc. Natl Acad. Sci. USA 107, 4275-4280 (2010).

34. De Henau, O. et al. Overcoming resistance to checkpoint blockade therapy by targeting PI3Kgamma in myeloid cells. Nature 539, 443-447 (2016).

35. Zamarin, D. et al. Intratumoral modulation of the inducible co-stimulator ICOS by recombinant oncolytic virus promotes systemic anti-tumour immunity. Nat. Commun. 8, 14340 (2017).

36. Kisielow, J., Obermair, F. J. \& Kopf, M. Deciphering CD4(+) T cell specificity using novel MHC-TCR chimeric receptors. Nat. Immunol. 20, 652-662 (2019).

37. Casey, A. E., Laster, W. R. Jr. \& Ross, G. L. Sustained enhanced growth of carcinoma EO771 in C57 black mice. Proc. Soc. Exp. Biol. Med. 77, 358-362 (1951).

38. Johnstone, C. N. et al. Functional and molecular characterisation of EO771. LMB tumours, a new C57BL/6-mouse-derived model of spontaneously metastatic mammary cancer. Dis. Model Mech. 8, 237-251 (2015).

39. van der Weyden, L. et al. Genome-wide in vivo screen identifies novel host regulators of metastatic colonization. Nature 541, 233-236 (2017).

40. Picelli, S. et al. Full-length RNA-seq from single cells using Smart-seq2. Nat Protoc. 9, 171-181 (2014).

41. Becht, E. et al. Dimensionality reduction for visualizing single-cell data using UMAP. Nat. Biotechnol. https://doi.org/10.1038/nbt.4314 (2018).

42. Martinez, F. O. \& Gordon, S. The M1 and M2 paradigm of macrophage activation: time for reassessment. F1000Prime Rep. 6, 13 (2014).

43. Roszer, T. Understanding the mysterious M2 macrophage through activation markers and effector mechanisms. Mediators Inflamm. 2015, 816460 (2015).

44. Desbarats, J., You-Ten, K. E. \& Lapp, W. S. Levels of p56lck and p59fyn are reduced by a glucocorticoid-dependent mechanism in graft-versus-host reaction-induced T cell anergy. Cell Immunol. 163, 10-18 (1995).

45. Van Laethem, F. et al. Glucocorticoids attenuate T cell receptor signaling. J. Exp. Med. 193, 803-814 (2001)

46. Heijink, I. H. \& Van Oosterhout, A. J. Strategies for targeting T-cells in allergic diseases and asthma. Pharmacol. Ther. 112, 489-500 (2006).

47. Gordon, J. R., Ma, Y., Churchman, L., Gordon, S. A. \& Dawicki, W Regulatory dendritic cells for immunotherapy in immunologic diseases. Front. Immunol. 5, 7 (2014).

48. Piemonti, L. et al. Glucocorticoids affect human dendritic cell differentiation and maturation. J. Immunol. 162, 6473-6481 (1999).

49. Woltman, A. M. et al. The effect of calcineurin inhibitors and corticosteroids on the differentiation of human dendritic cells. Eur. J. Immunol. 30 1807-1812 (2000)

50. Chen, X., Oppenheim, J. J., Winkler-Pickett, R. T., Ortaldo, J. R. \& Howard, O. M. Glucocorticoid amplifies IL-2-dependent expansion of functional FoxP3(+)CD4(+)CD25(+) T regulatory cells in vivo and enhances their capacity to suppress EAE. Eur. J. Immunol. 36, 2139-2149 (2006).

51. $\mathrm{Hu}, \mathrm{Y}$. et al. Function of regulatory T-cells improved by dexamethasone in Graves' disease. Eur. J. Endocrinol. 166, 641-646 (2012).

52. Suarez, A., Lopez, P., Gomez, J. \& Gutierrez, C. Enrichment of CD4+ CD25high $\mathrm{T}$ cell population in patients with systemic lupus erythematosus treated with glucocorticoids. Ann. Rheum. Dis. 65, 1512-1517 (2006).

53. Anderson, A. C., Joller, N. \& Kuchroo, V. K. Lag-3, Tim-3, and TIGIT: coinhibitory receptors with specialized functions in immune regulation. Immunity 44, 989-1004 (2016).

54. Rhen, T. \& Cidlowski, J. A. Antiinflammatory action of glucocorticoids-new mechanisms for old drugs. New Engl. J. Med. 353, 1711-1723 (2005).

55. Flint, T. R. et al. Tumor-induced IL-6 reprograms host metabolism to suppress anti-tumor immunity. Cell Metab. 24, 672-684 (2016).

56. Connell, C. M. et al. Cancer immunotherapy trial registrations increase exponentially but chronic immunosuppressive glucocorticoid therapy may compromise outcomes. Ann. Oncol. 28, 1678-1679 (2017).

57. Della Corte, C. M. \& Morgillo, F. Early use of steroids affects immune cells and impairs immunotherapy efficacy. ESMO Open 4, e000477 (2019). 
58. Draghi, A. et al. Differential effects of corticosteroids and anti-TNF on tumorspecific immune responses: implications for the management of irAEs. Int. J. Cancer 145, 1408-1413 (2019).

59. Obradovic, M. M. S. et al. Glucocorticoids promote breast cancer metastasis. Nature 567, 540-544 (2019).

60. Giles, A. J. et al. Dexamethasone-induced immunosuppression: mechanisms and implications for immunotherapy. J. Immunother. Cancer 6, 51 (2018).

61. Arbour, K. C. et al. Impact of baseline steroids on efficacy of programmed cell death-1 and programmed death-ligand 1 blockade in patients with non-smallcell lung cancer. J. Clin. Oncol. 36, 2872-2878 (2018).

62. Tokunaga, A. et al. Selective inhibition of low-affinity memory CD8(+) T cells by corticosteroids. J. Exp. Med. https://doi.org/10.1084/jem.20190738 (2019).

63. Hodgkins, A. et al. WGE: a CRISPR database for genome engineering. Bioinformatics 31, 3078-3080 (2015).

64. Kranz, A. et al. An improved Flp deleter mouse in $\mathrm{C} 57 \mathrm{Bl} / 6$ based on Flpo recombinase. Genesis 48, 512-520 (2010).

65. Pramanik, J. et al. Genome-wide analyses reveal the IRE1a-XBP1 pathway promotes $\mathrm{T}$ helper cell differentiation by resolving secretory stress and accelerating proliferation. Genome Med. 10, 76 (2018).

66. Buenrostro, J. D., Giresi, P. G., Zaba, L. C., Chang, H. Y. \& Greenleaf, W. J. Transposition of native chromatin for fast and sensitive epigenomic profiling of open chromatin, DNA-binding proteins and nucleosome position. Nat. Methods 10, 1213-1218 (2013).

67. Langmead, B. \& Salzberg, S. L. Fast gapped-read alignment with Bowtie 2. Nat. Methods 9, 357-359 (2012).

68. Zhang, Y. et al. Model-based analysis of ChIP-Seq (MACS). Genome Biol. 9, R137 (2008).

69. Kent, W. J. et al. The human genome browser at UCSC. Genome Res. 12 996-1006 (2002).

70. Wei, G. et al. Genome-wide analyses of transcription factor GATA3-mediated gene regulation in distinct T cell types. Immunity 35, 299-311 (2011).

71. Bogunovic, D. et al. Immune profile and mitotic index of metastatic melanoma lesions enhance clinical staging in predicting patient survival. Proc. Natl Acad. Sci. USA 106, 20429-20434 (2009).

72. Linnemann, C. et al. High-throughput epitope discovery reveals frequent recognition of neo-antigens by $\mathrm{CD} 4+\mathrm{T}$ cells in human melanoma. Nat. Med. 21, 81-85 (2015)

\section{Acknowledgements}

The study was supported by CRUK Cancer Immunology fund (Ref. 20193), ERC consolidator grant (ThDEFINE, Project ID: 646794) and Wellcome Sanger Institute core funding (WT206194). We are thankful to the funders. We would like to thank Ana C. Anderson and Rahul Roychoudhuri for their valuable comments on the manuscript and useful discussions; Sarah Davidson and Emma Mahata for their help with diagram illustration; Bee Ling Ng, Chris Hall, Sam Thompson, and Jennie Graham for help with flow cytometry and cell sorting; Research Support Facility, WSI, for their technical help and animal husbandry. EO771 cells, hU6 expression plasmid, C57BL/6 JM8 ESC cells, and pCAGGSs-Flpo, were kindly gifted by Robin Anderson, Sebastian Gerety, Bill Skarnes, and Andrea Kranz, respectively. We are thankful to them.

\section{Author contributions}

B.M.: led and managed the project, generated hypothesis, designed and performed experiments, analyzed data, and wrote manuscript J.P.: performed experiments, analyzed data, and helped in genetically modified mouse generation. L.v.d.W.: performed B16-F10 and EO771 pulmonary metastasis experiments, analyzed data. K.P.: analyzed single-cell RNA-Seq data. A.R.: performed B16-F10 subcutaneous tumors experiments, analyzed data. G.K., N.A.F., and K.K.: analyzed publicly available gene expression datasets to confirm human tumor expression of steroidogenic genes. X.C.: performed and analyzed ATAC-seq and ChIP-seq data. L.S.C.: helped in identification and quantification of tumor spots in EO771 lung metastasis. E.R. and G.D.: helped in generation of Cyp11amCherry and Cyp111a ${ }^{f l / f l}$ mouse model. IW: built the Cyp11a1-mCherry targeting construct. K.O.: helped in designing experiments, writing manuscript, critical comments and supervision. D.J.A.: conducted pulmonary metastasis experiments. J.S.: conducted B16-F10 subcutaneous experiments under her PPL and supervised the study. S.A.T.: supervised the study. All authors commented on and approved of the draft manuscript before submission.

\section{Competing interests}

In the last three years, S.A.T has consulted for Genentech and Roche, and is a member of Scientific Advisory Boards at Biogen, GlaxoSmithKline and Foresite Labs. The remaining authors declare no competing interests.

\section{Additional information}

Supplementary information is available for this paper at https://doi.org/10.1038/s41467020-17339-6.

Correspondence and requests for materials should be addressed to B.M., J.D.S. or S.A.T.

Peer review information Nature Communications thanks the anonymous reviewers for their contribution to the peer review of this work.

Reprints and permission information is available at http://www.nature.com/reprints

Publisher's note Springer Nature remains neutral with regard to jurisdictional claims in published maps and institutional affiliations.

(c) (i) Open Access This article is licensed under a Creative Commons Attribution 4.0 International License, which permits use, sharing, adaptation, distribution and reproduction in any medium or format, as long as you give appropriate credit to the original author(s) and the source, provide a link to the Creative Commons license, and indicate if changes were made. The images or other third party material in this article are included in the article's Creative Commons license, unless indicated otherwise in a credit line to the material. If material is not included in the article's Creative Commons license and your intended use is not permitted by statutory regulation or exceeds the permitted use, you will need to obtain permission directly from the copyright holder. To view a copy of this license, visit http://creativecommons.org/ licenses/by/4.0/.

(C) The Author(s) 2020 